\title{
Greenhouse gas emission in constructed wetlands for wastewater treatment: A review
}

\author{
Ülo Mander ${ }^{\mathrm{a}, \mathrm{b}, *}$, Gabriela Dotro $^{\mathrm{c}}$, Yoshitaka Ebie ${ }^{\mathrm{d}}$, Sirintornthep Towprayoon ${ }^{\mathrm{e}}$, \\ Chart Chiemchaisri ${ }^{\mathrm{f}}$, Sandra Furlan Nogueira ${ }^{\mathrm{g}}$, Baasansuren Jamsranjav ${ }^{\mathrm{h}}$, \\ Kuno Kasak ${ }^{\mathrm{a}}$, Jaak Truu ${ }^{\mathrm{a}}$, Julien Tournebize ${ }^{\mathrm{b}}$, William J. Mitsch ${ }^{\mathrm{i}}$ \\ a Department of Geography, Institute of Ecology and Earth Sciences, University of Tartu, 46 Vanemuise Street, 51014 Tartu, Estonia \\ ${ }^{\mathrm{b}}$ Hydrosystems and Bioprocesses Research Unit, National Research Institute of Science and Technology for Environment and Agriculture (Irstea), \\ 1 rue Pierre-Gilles de Gennes CS 10030, F92761 Antony Cedex, France \\ ${ }^{c}$ Cranfield Water Science Institute, School of Applied Sciences, Cranfield University, College Road, Cranfield, Bedfordshire MK43 0AL, United Kingdom \\ ${ }^{\mathrm{d}}$ Center for Material Cycles and Waste Management Research, National Institute for Environmental Studies, Ibaraki 3058506, Japan \\ e Joint Graduate School of Energy and Environment, King Mongkut's University of Technology, Thonburi, 126 Pracha-utid Road, Bangmod Toongkru, \\ Bangkok 10140 Thailand \\ ${ }^{\mathrm{f}}$ Department of Environmental Engineering, Faculty of Engineering, Kasetsart University, 50 Ngam Wong Wan Road, Bangkok 10900, Thailand \\ ${ }^{\mathrm{g}}$ Embrapa Monitoramento por Satélite, Av. Soldado Passarinho, 303, 13070-115 Campinas, SP, Brazil \\ h Technical Support Unit, Intergovernmental Panel on Climate Change (IPCC) Task Force on National Greenhouse Gas Inventories, C/o Institute for Global \\ Environmental Strategies, 2108-11, Kamiyamaguchi, Hayama, Kanagawa 240-0115, Japan \\ ${ }^{\mathrm{i}}$ Everglades Wetland Research Park, Florida Gulf Coast University, 4940 Bayshore Drive, Naples, FL 34112, USA
}

\section{A R T I C L E I N F O}

\section{Article history:}

Received 11 July 2013

Received in revised form 9 December 2013

Accepted 17 December 2013

Available online $\mathrm{xxx}$

\section{Keywords:}

Carbon dioxide

HSSF

Intermittent loading

Methane

Nitrous oxide

Pulsing hydrology

Surface flow constructed wetlands

VSSF

\begin{abstract}
A B S T R A C T
A literature analysis of 158 papers published in international peer-reviewed journals indexed by the Thomson Reuters Web of Knowledge from 1994 to 2013 showed that $\mathrm{CO}_{2}-\mathrm{C}$ emission was significantly lower in free water surface (FWS) constructed wetlands (CW) than in subsurface flow (SF) CWs (median values from 95.8 to $137.0 \mathrm{mg} \mathrm{m}^{-2} \mathrm{~h}^{-1}$, respectively). In vertical subsurface flow (VSSF) $\mathrm{CWs}$ the $\mathrm{CH}_{4}-\mathrm{C}$ emission was significantly lower than in horizontal subsurface flow (HSSF) CWs (median values 3.0, 6.4, and $4.0 \mathrm{mg} \mathrm{m}^{-2} \mathrm{~h}^{-1}$, respectively). There were no significant differences in $\mathrm{N}_{2} \mathrm{O}-\mathrm{N}$ emission in various CW types (median for FWS, VSSF and HSSF CWs: $0.09,0.12$, and $0.13 \mathrm{mg} \mathrm{m}^{-2} \mathrm{~h}^{-1}$ correspondingly).

The highest value of emission factor (EF) of $\mathrm{CH}_{4}\left(\left(\mathrm{CH}_{4}-\mathrm{C} / \text { inflow } \mathrm{TOC}_{\text {in }}\right)^{*} 100 \%\right)$ was found for FWS CWs (median 18.0\%), followed by HSSF CWs (3.8\%), and VSSF CWs (1.28\%). Median values of $\mathrm{N}_{2} \mathrm{O}$ EFs $\left(\left(\mathrm{N}_{2} \mathrm{O}-\mathrm{N} / \text { inflow } \mathrm{TN}_{\text {in }}\right)^{*} 100 \%\right)$ differed significantly in all three CW types: $0.34 \%$ for HSSF, $0.11 \%$ for FWS, and $0.018 \%$ for VSSF CWs.

We found a significant correlation between $\mathrm{TOC}_{\text {in }}$ and $\mathrm{CH}_{4}-\mathrm{C}$ emission and between the $\mathrm{TN}_{\text {in }}$ and $\mathrm{N}_{2} \mathrm{O}-\mathrm{N}$ emission values for all of the types of CWs we studied.

Hybrid CWs (e.g., the subsequent combination of VSSF, HSSF and FWS CWs) are beneficial from the point of view of both water purification and minimization of greenhouse gas (GHG) emissions. Likewise, intermittent loading in VSSF CWs and macrophyte harvesting in HSSF and FWS CWs can mitigate GHG emissions.
\end{abstract}

(c) 2013 Elsevier B.V. All rights reserved.

\section{Introduction}

Constructed wetlands (CW) are engineered wetland systems that have been designed and constructed to utilize natural processes in treating wastewater (Vymazal et al., 1998). Constructed

\footnotetext{
* Corresponding author at: Department of Geography, Institute of Ecology and Earth Sciences, University of Tartu, 46 Vanemuise Street, 51014 Tartu, Estonia. Tel.: +372 7375816 ; fax: +372 7375825 .

E-mail address: ulo.mander@ut.ee (Ü. Mander).
}

wetlands are used to improve the quality of wastewater from point and nonpoint sources of water pollution, including domestic, industrial and municipal wastewater, stormwater runoff, farm wastewater, collated runoff from agricultural land and landfill leachate (Kadlec and Knight, 1996; Kadlec and Wallace, 2008).

The main types of CWs are: free water surface (FWS) or surface flow, vertical subsurface flow (VSSF) and horizontal subsurface flow (HSSF) CWs (Vymazal, 2007, 2011). In addition to wastewater treatment, the CWs provide several ecosystem services such as provisional (food, energy, fibers), regulating (carbon (C) sequestration, climate regulating, flood control), supporting (biodiversity,

0925-8574/\$ - see front matter (C) 2013 Elsevier B.V. All rights reserved. http://dx.doi.org/10.1016/j.ecoleng.2013.12.006 
nutrient cycling) and cultural (recreational, educational) services (Mitsch and Gosselink, 2007).

Free water surface CWs are shallow and low flow velocity wetlands which have areas of open water and floating, submerged and/or emergent plants (Kadlec and Wallace, 2008). FWS CWs are very effective in the removal of organics through microbial degradation and the removal of suspended solids through filtration and sedimentation (Vymazal et al., 1998). The removal of nitrogen (N) and phosphorus (P) can be sustainable, but depends on inflow concentration, the chemical form of nitrogen, water temperature, the season, organic carbon availability, substrate material and dissolved oxygen concentration (Vymazal, 2011). The FWS wetlands are mostly used for the tertiary treatment of domestic and municipal wastewater, mine drainage waters, and for stormwater and agricultural runoff (Kadlec and Knight, 1996; Kadlec and Wallace, 2008).

In horizontal subsurface flow CWs, the wastewater is fed in at the inlet and flows slowly through the porous medium under the surface of the bed planted with emergent vegetation to the outlet, where it is collected before leaving via a water level control structure (Vymazal et al., 1998). During passage the wastewater comes into contact with a network of aerobic, anoxic and anaerobic zones. Most of the bed is anoxic/anaerobic due to the permanent saturation of the beds. The aerobic zones occur around roots and rhizomes that leak oxygen into the substrate (Brix, 1987). The most important properties of macrophytes planted in HSSF CWs are filtration bed insulation during the winter, substrate for the growth of attached bacteria, oxygen release to the rhizosphere, nutrient uptake and storage, $C$ sequestration and root exudates with antimicrobial properties (Brix, 1997; Vymazal and Kröpfelova, 2008). HSSF CWs are commonly sealed with a liner to prevent seepage and to ensure controllable outflow, and are mostly used for secondary treatment of domestic and municipal wastewater (Vymazal and Kröpfelova, 2008). Organic compounds are degraded by bacteria under aerobic and anaerobic conditions. It has been shown that the oxygen transport capacity in these systems is insufficient to ensure aerobic decomposition and that anaerobic processes play an important role in HSSF CWs (Vymazal and Kröpfelova, 2008). Suspended solids settle into micropockets in the filtration bed or are filtered out. Removal of ammonia- $\mathrm{N}$ is limited by the lack of oxygen and hence nitrification in the filtration media. The HSSF CWs do, however, provide suitable conditions for denitrification (Vymazal and Kröpfelova, 2008). Removal of P is usually low unless special media with high sorption capacity are used. The selection of filtration material is also very important for the longevity of the system, because media that are too fine will clog the system, and surface runoff will occur (Vohla et al., 2011).

Vertical subsurface flow CWs comprise a flat bed of graded gravel topped with sand or other porous filter materials planted with macrophytes. In contrast to HSSF CWs, VSSF CWs are fed intermittently with large batches, thus flooding the surface. Wastewater then percolates down through the bed and is collected by a drainage network at the bottom. The bed drains completely, which allows air to refill the bed. The VSSF CWs provide greater oxygen transfer into the bed, thus producing a nitrified (high $\mathrm{NO}_{3}{ }^{-}$) effluent (Cooper et al., 1996; Cooper, 2005). On the other hand, VSSF CWs do not provide suitable conditions for denitrification to complete conversion to gaseous nitrogen forms which escape to the atmosphere. Removal of organics and suspended solids is high (Vymazal and Kröpfelova, 2008). As compared to HSSF CWs, which need 5-6 $\mathrm{m}^{2}$ per population equivalent $(\mathrm{PE})$, vertical flow systems require less land, usually 1-3 $\mathrm{m}^{2} \mathrm{PE}^{-1}$ (Cooper, 2005).

Both VSSF and HSSF CWs with the ability to insulate the surface of the bed are capable of operation under colder conditions than are FWS systems (Mander and Jenssen, 2003).
Various types of CWs are usually combined (i.e., hybrid or combined systems) in order to achieve higher removal efficiency, especially for nitrogen. The design commonly consists of two stages: several parallel VSSF beds followed by two or three HSSF beds in series (Vymazal, 2007). The VSSF wetland is intended to remove organics and suspended solids and to provide nitrification, while denitrification and the further removal of organics and suspended solids occur in the HSSF wetland. When aquatic macrophyte production is the main practical function of a wetland system, the VSSF-HSSF bed complex can be followed by a larger FWS wetland (Maddison et al., 2009).

As a bias of the water purification, the CWs for wastewater treatment have been found to be sources of greenhouse gases (GHG). Carbon dioxide $\left(\mathrm{CO}_{2}\right)$ emission has been measured in few full-scale CWs (Mander et al., 2003, 2005a,b, 2008; Teiter and Mander, 2005; Liikanen et al., 2006; Ström et al., 2006; Garcia et al., 2007; Picek et al., 2007; Van der Zaag et al., 2010), and C balance has been compiled in only one HSSF CW based on the long-term direct measurement of $C$ in inflow and outflow, accumulation in filter material (sand), microbes, above ground and below ground plant biomass, and the emission of $\mathrm{CO}_{2}$ and $\mathrm{CH}_{4}$ (Mander et al., 2008). On the other hand, there are more measurements of $\mathrm{CH}_{4}$ and $\mathrm{N}_{2} \mathrm{O}$ emission from full-scale CWs: $\mathrm{CH}_{4}$ by Tanner et al. (1997), Xue et al. (1999), Johansson et al. (2004), and Chiemchaisri et al. (2008); $\mathrm{N}_{2} \mathrm{O}$ by Fey et al. (1999) and Johansson et al. (2003); and both $\mathrm{CH}_{4}$ and $\mathrm{N}_{2} \mathrm{O}$ by Tai et al. (2002), Wild et al. (2001), Mander et al. (2003, 2005a,b, 2008, 2011), Stadmark and Leonardson (2005), Teiter and Mander (2005), Liikanen et al. (2006), Søvik et al. (2006), Gui et al. (2007), Picek et al. (2007), Søvik and Kløve (2007), Ström et al. (2006), Liu et al. (2009), and Van der Zaag et al. (2010).

Recent research has shown that $\mathrm{N}_{2} \mathrm{O}$ can be produced through a number of different pathways, both chemical and biochemical, during nitrification (stepwise conversion of ammonia to nitrate) and denitrification (stepwise conversion of nitrate to nitrogen gas; Colliver and Stephenson, 2000). Under aerobic conditions in a nitrifying wastewater treatment system, $\mathrm{N}_{2} \mathrm{O}$ production through nitrifier denitrification has been identified as the predominant production pathway (Wunderlin et al., 2013; Aboobakar et al., 2013). Similarly, research from soil science has shown that in wellaerated, moist conditions (soil water filled pore space at $40-60 \%$ ), $\mathrm{N}_{2} \mathrm{O}$ can be emitted during nitrification (Robertson and Tiedje, 1987; Mosier, 1998; Mosier et al., 1998) by ammonia-oxidizing bacteria during the oxidation of hydroxylamine $\left(\mathrm{NH}_{2} \mathrm{OH}\right)$ to nitrite $\left(\mathrm{NO}_{2}{ }^{-}\right.$) (Arp and Stein, 2003), and also via reducing $\mathrm{NO}_{2}{ }^{-}$to $\mathrm{N}_{2} \mathrm{O}$ and $\mathrm{N}_{2}$ under aerobic conditions by nitrifier denitrification (Goreau et al., 1980; Wrage et al., 2001).

Denitrification, as the microbial reduction of $\mathrm{NO}_{3}-\mathrm{N}$ to $\mathrm{NO}_{2}-\mathrm{N}$ and further to gaseous forms of $\mathrm{NO}, \mathrm{N}_{2} \mathrm{O}$ and $\mathrm{N}_{2}$ (Knowles, 1982), has been found in numerous studies to be a significant process in nitrogen removal in treatment wetlands (Bachand and Horne, 2000a,b; Spieles and Mitsch, 2000; Hernandez and Mitsch, 2006, 2007; Batson et al., 2012). Denitrification rates in soils are influenced by nitrate availability, carbon availability, temperature and $\mathrm{pH}$ (Mitsch and Gosselink, 2007). The last step of denitrification, i.e., the conversion of $\mathrm{N}_{2} \mathrm{O}$ to $\mathrm{N}_{2}$, is very sensitive to oxygen and redox status, and disruption of this step results in incomplete denitrification and $\mathrm{N}_{2} \mathrm{O}$ emissions (Colliver and Stephenson, 2000). The relative contribution to $\mathrm{N}_{2} \mathrm{O}$ emissions from a treatment system will depend on the environmental conditions that are generated and maintained throughout the pollutant transformation processes. Both denitrification and methane formation depend on the oxygen and redox status of the soil or sediment, which changes in both spatial and temporal contexts. In this relation, the variability of fluxes of both $\mathrm{N}_{2} \mathrm{O}$ and $\mathrm{CH}_{4}$ is high (Willison et al., 1998; Teiter and Mander, 2005). 


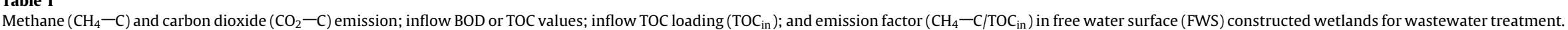

Average values for each site/event are presented. Climate zones: T - temperate, B - boreal, W - warm, sTr - subtropical, n.a. - not available.

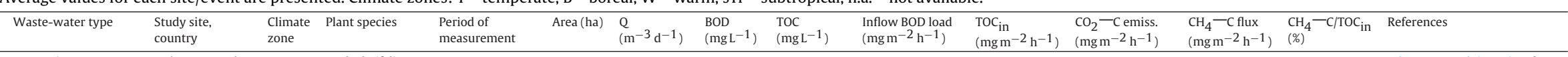

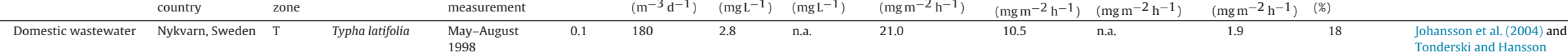
1998
April-October
1999 Domestic wastewater Nykvarn, Sweden $\quad \mathrm{T} \quad$ Phalaris

Domestic wastewater Nykvarn, Sweden T Glycria maxima Domestic wastewater Nykvarn, Sweden $\quad \mathrm{T} \quad$ Lemna minor Domestic wastewater Nykvarn, Sweden $\quad \mathrm{T} \quad$ Spirogyra spp. Domestic wastewater Nytwan Sweden T- Plots without - Mag$\begin{array}{lll}\text { Domestic wastewater Nykvarn, Sweden } & \mathrm{T} & \begin{array}{l}\text { Plots without } \\ \text { plants }\end{array}\end{array}$ 1999 $\begin{array}{cccl}\text { Domestic wastewater } & \text { Lakeus, Finland } & \text { B } & \begin{array}{l}\text { Phragmites } \\ \text { australis, T. } \\ \text { latifolia } \\ \text { Carex-Sphagnum }\end{array} \\ \text { Domestic wastewater } & \text { Ruka, Finland } & \text { B }\end{array}$ $\begin{array}{lllll}\text { Domestic wastewater } & \text { Skallstuggu, } & \text { B } & \text { P. australis } & 2002 \\ & \text { Norway } & & & \text { July } \\ 2001-\end{array}$ Domestic wastewater Skjønhaug $\quad$ B $\quad$ Iris pseudacorus, July

$\begin{array}{lll}\text { Domestic wastewater } & \begin{array}{l}\text { Magle, } \\ \text { Hässleholm, }\end{array} \quad \mathrm{T} \quad \begin{array}{l}\text { lacustris } \\ \text { Juncus effusus, } \\ \text { latifolia, } P .\end{array}\end{array}$

Sweden australis

$\begin{array}{clcl}\text { Domestic wastewater } & \begin{array}{l}\text { Skjonhaug } \\ \text { Norway }\end{array} & \text { T } & \begin{array}{l}\text { I. pseudacorus. T. } \\ \text { latifolia, S. } \\ \text { lacustris }\end{array} \\ \text { P. australis }\end{array}$

Domestic wastewater Miho, Ibaraki, $\quad \mathrm{T} / \mathrm{W} \quad$ P. australis

Domestic wastewater Jiho

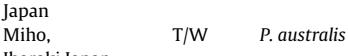

Agricult. non-point $\quad$ Donau-moos,

$\begin{array}{ll}\text { pollution } & \text { Donau-mo } \\ \text { Germany } & \end{array}$

$\begin{array}{llll}\begin{array}{cl}\text { Agricult. non-point } \\ \text { pollution }\end{array} & \begin{array}{l}\text { Donau-moos, } \\ \text { Germany }\end{array} & \text { T } & \begin{array}{l}\text { angusin 2,T. } \\ \text { latifolia, } T \text {. }\end{array}\end{array}$

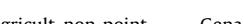

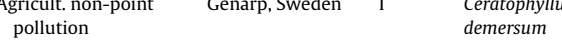

Agricult. non-point $\quad$ Görarp, Sweden $\quad T$ May-Augus 1998 April-October
1999 May-August 1998 pril-October inor May-A 1998
April-October 1999 May-Augu 1998 April-October April-October
1999 May-August 1998 April-October nuary $\begin{array}{ll}\text { T. latifolia, } & 2001-\mathrm{F} \\ \text { Schoenoplectus } & 2002\end{array}$ Schoenoplectus 2002

Basin 1: T.

latifolia, T.
angustifolic

\section{$0.1 \quad 180$}

$0.09 \quad 18$

$0.09 \quad 18$

$0.09 \quad 180$

$0.09 \quad 18$

$0.0065 \quad$ n.a.

(a)

01-February

April-May 2005

October

2001-July 2002

August

2004-May 2006

June-Augu

September

1998-May 1999

September

September
1998-May 1999

Ju

No plants in plots

$$
2004
$$

$$
\begin{aligned}
& \text { June } 2003-M \\
& 2004
\end{aligned}
$$

June 2003-May

2004

$0.7 \quad 3500$

$0.4 \quad 600$

$20 \quad 13,0$

$\begin{array}{ll}0.4 \quad 5 \\ -0.0012 & 0.5\end{array}$

$0.0012 \quad 0.5$

2.2

4286

Agricult. non-point $\quad$ Ormastorp, $\quad$ T $\quad$ L. minor

$\begin{array}{llll}5.2 & \text { n.a. } \quad 39.0 & 19.5\end{array}$

19.5

n.a.

$2.2 \quad$ n.a.

18.3

\begin{tabular}{ll}
$0.0012 \quad 0.5$ \\
\hline
\end{tabular}

$2.6 \quad 158$

15,000

\begin{tabular}{|c|c|c|c|c|}
\hline 8.2 & n.a. & 68.3 & 34.2 & n.a. \\
\hline 2.7 & n.a. & 22.5 & 11.3 & n.a. \\
\hline 3.3 & n.a. & 27.5 & 13.8 & n.a. \\
\hline n.a. & 25 & n.a. & 85.8 & 108.3 \\
\hline n.a. & 17 & n.a. & 25.0 & 95.8 \\
\hline n.a. & n.a. & n.a. & 22.0 & 87.5 \\
\hline n.a. & 26.7 & n.a. & 166.9 & 49.6 \\
\hline 19 & n.a. & 51.5 & 25.7 & 100 \\
\hline 15 & n.a. & 81.3 & 40.6 & n.a. \\
\hline 107.3 & 10.3 & n.a. & 17.9 & n.a. \\
\hline 200 & n.a. & 347.2 & 173.6 & n.a. \\
\hline n.a. & 3.5 & n.a. & 1.0 & n.a. \\
\hline n.a. & 11.2 & n.a. & 2.8 & n.a. \\
\hline n.a. & 1.5 & n.a. & 26.8 & n.a. \\
\hline n.a. & 1.3 & n.a. & 54.2 & n.a. \\
\hline n.a. & 1.8 & n.a. & 37.5 & n.a. \\
\hline
\end{tabular}

(350

Johnderski and Hansson
(2001) for BOD data

Johansson et al. (2004) and Tonderski and Hansson (2001) for BOD data

Johansson et al. (2004) and (2001) for BOD data

Johansson et al (2004) and Tonderski and Hansso (2001) for BOD data

Johansson et al. (2004) and (2001) for BOD data

Johansson et al. (2004) and Tonderski and Hansso

(2001) for BOD data

Søvik et al. (2006)

Søvik et al. (2006)

Søvik et al. (2006)

Søvik et al. (2006)

Ström et al. (2006); msvattense

$\begin{array}{lll}181.0^{\text {a }} & 446^{\text {a }} & \begin{array}{l}\text { for BOD data } \\ \text { Søvik and Kløve (2007) }\end{array}\end{array}$

Gui et al. (2007)

Liu et al. (2009)

Wild et al. (2001)

Wild et al. (2001)

Stadmark and Leonardso (2005); Swedish River

Stadmark and Leonardson

(2005); Swedish River

Inventory for TOC data

Stadmark and Leonards

(2005); Swedish River
Inventory for TOC data

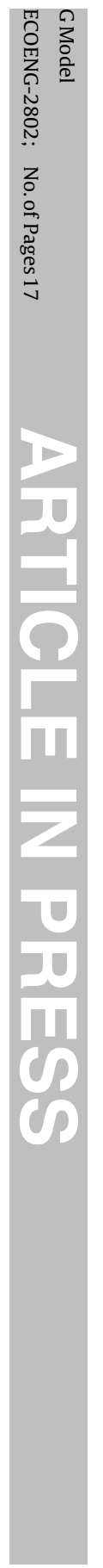




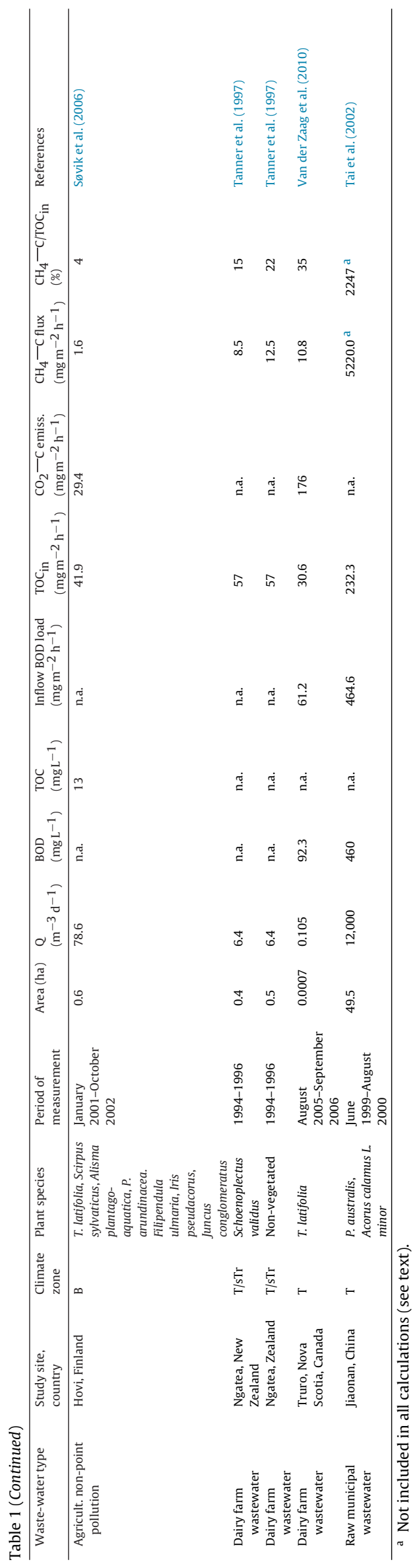

Methane is produced in anoxic soils and sediments, while welldrained soils act as a sink for atmospheric $\mathrm{CH}_{4}$ due to methane oxidation, through either ammonia oxidizers or methanotrophs (Le Mer and Roger, 2001). In treatment systems, such as wetlands, methane is generated from the anaerobic degradation of organic matter either present in the influent to the systems or accumulated as a result of plant litter accumulation. Methane generation occurs in the oxidation-reduction state range of -250 to $-350 \mathrm{mV}$ (Mitsch and Gosselink, 2007), in the absence of oxygen. In some conditions, it can start already at $-150 \mathrm{mV}$ (Wang et al., 1993). As such, emissions of methane from treatment wetlands are expected to be higher in anaerobic systems such as HSSF systems and, to a lesser extent, FWS and organically overloaded systems.

Due to increasing human impact on the global environment, nitrous oxide, which has an atmospheric lifetime of about 120 years, a global warming potential of 296 relative to $\mathrm{CO}_{2}$ over a 100-year time horizon, and is responsible for about $6 \%$ of anticipated warming (IPCC, 2007), is increasing in the atmosphere at a rate of about $0.3 \% \mathrm{yr}^{-1}$. Methane in the atmosphere has a lifetime of 8.4 years on a 100-year time horizon, $\mathrm{CH}_{4}$ has a global warming potential of 25 relative to $\mathrm{CO}_{2}$, and is responsible for about $20 \%$ of anticipated warming (IPCC 2007).

The main objectives of the study are: (1) to give an overview of GHG emissions and the main influencing factors in all types of CWs; (2) to analyze the relationship between $\mathrm{CH}_{4}$ and $\mathrm{N}_{2} \mathrm{O}$ emission and related $\mathrm{C}$ and $\mathrm{N}$ loading in the inflow of $\mathrm{CW}$ systems; (3) to determine the emission factor (EF) values of $\mathrm{CH}_{4}$ and $\mathrm{N}_{2} \mathrm{O}$ for different types of CWs.

Recently published reviews on $\mathrm{N}_{2} \mathrm{O}$ emission from CWs (Huang et al., 2013) and on climate regulation by FWS and created riverine wetlands (Mander et al., 2014) cover only part of CWs and a fraction of the available literature sources, whereas this paper seeks to cover the entire topic.

\section{Materials and methods}

\subsection{Data sources and analysis}

We reviewed 158 papers published in international peerreviewed journals indexed by the Thomson Reuters Web of Knowledge from 1994 to 2013. The terms "free water surface", "surface flow", constructed wetland(s)", "artificial wetland(s)", "treatment wetland(s)", "subsurface flow wetland(s)", "vertical subsurface flow" and "horizontal subsurface flow" in combination with the terms "carbon dioxide", " $\mathrm{CO}_{2}$ ", "methane", " $\mathrm{CH}_{4}$ ", "nitrous oxide" and " $\mathrm{N}_{2} \mathrm{O}$ " were searched.

We found a total of 14 publications that provided information on emissions of either $\mathrm{CH}_{4}$ or $\mathrm{N}_{2} \mathrm{O}\left(\mathrm{g} \mathrm{m}^{-2} \mathrm{~h}^{-1}\right)$ or both gases in FWS CWs. These publications presented information on 19 different SF CW systems, whereas for $\mathrm{CH}_{4}$ and $\mathrm{N}_{2} \mathrm{O}$ there were 25 and 26 subsystems/measuring events, respectively, from which EF values could be calculated (Tables 1-6).

Regarding the vertical subsurface flow (VSSF) CWs, there were only 4 measurement periods presented for $3 \mathrm{CWs}$ from which $\mathrm{CH}_{4}$ emission data and EFs could be calculated: Kõo in Estonia (Teiter and Mander, 2005; Søvik et al., 2006), Ski in Norway (Søvik et al., 2006), and Miho/Ibaraki, Japan (Gui et al., 2007; Liu et al., 2009; Table 3). For $\mathrm{N}_{2} \mathrm{O}$ emissions, laboratory microcosm experiments with different plant species from Ibaraki, Japan (Inamori et al., 2008; Wang et al., 2008a,b) were included (Table 4).

Regarding $\mathrm{CH}_{4}$ fluxes from HSSF CWs (Table 5) we possessed data from 9 systems. For $\mathrm{N}_{2} \mathrm{O}$ emissions from HSSFs, a CW for dairy 
Table 2

Nitrous oxide $\left(\mathrm{N}_{2} \mathrm{O}-\mathrm{N}\right)$ emission; inflow total nitrogen loading $\left(\mathrm{TN}_{\mathrm{in}}\right)$; and emission factor $\left(\mathrm{N}_{2} \mathrm{O}-\mathrm{N} / \mathrm{TN}_{\text {in }}\right)$ in free water surface (FWS) constructed wetlands for wastewater treatment. Average values for each site/event are presented. Climate zones: $\mathrm{T}$ - temperate, $\mathrm{B}$ - boreal, $\mathrm{W}$ - warm, n.a. - not available.

\begin{tabular}{|c|c|c|c|c|c|c|c|c|c|c|c|}
\hline Wastewater type & Site, country & $\begin{array}{l}\text { Climate } \\
\text { zone }\end{array}$ & Plant species & $\begin{array}{l}\text { Period of } \\
\text { measurement }\end{array}$ & Area (ha) & $Q\left(m^{-3} d^{-1}\right)$ & $\mathrm{TN}\left(\mathrm{mg} \mathrm{L}^{-1}\right)$ & $\begin{array}{l}\mathrm{TN}_{\text {in }}\left(\mathrm{mg} \mathrm{m}^{-2}\right. \\
\left.\mathrm{h}^{-1}\right)\end{array}$ & $\begin{array}{l}\mathrm{N}_{2} \mathrm{O} \text { flux } \\
\left(\mathrm{mg} \mathrm{m}^{-2} \mathrm{~h}^{-1}\right)\end{array}$ & $\mathrm{N}_{2} \mathrm{O}-\mathrm{N} / \mathrm{TN}_{\text {in }}(\%)$ & References \\
\hline Domestic wastewater & Nykvarn, Sweden & $\mathrm{T}$ & T. latifolia & $\begin{array}{l}\text { May-August } 1998 \\
\text { April-October } 1999\end{array}$ & 0.1 & 180 & 8 & 60 & 0.081 & 0.14 & Johansson et al. (2003) \\
\hline Domestic wastewater & Nykvarn, Sweden & $\mathrm{T}$ & P. arundinacea & $\begin{array}{l}\text { May-August } 1998 \\
\text { April-October } 1999\end{array}$ & 0.1 & 180 & 8 & 60 & 0.152 & 0.25 & Johansson et al. (2003) \\
\hline Domestic wastewater & Nykvarn, Sweden & $\mathrm{T}$ & Glyceria maxima & $\begin{array}{l}\text { May-August } 1998 \\
\text { April-October } 1999\end{array}$ & 0.09 & 180 & 8 & 67 & 0.031 & 0.05 & Johansson et al. (2003) \\
\hline Domestic wastewater & Nykvarn, Sweden & $\mathrm{T}$ & L. minor & $\begin{array}{l}\text { May-August } 1998 \\
\text { April-October } 1999\end{array}$ & 0.09 & 180 & 8 & 67 & 0.094 & 0.14 & Johansson et al. (2003) \\
\hline Domestic wastewater & Nykvarn, Sweden & $\mathrm{T}$ & Spirogyra spp. & $\begin{array}{l}\text { May-August } 1998 \\
\text { April-October } 1999\end{array}$ & 0.09 & 180 & 8 & 67 & 0.036 & 0.05 & Johansson et al. (2003) \\
\hline Domestic wastewater & Nykvarn, Sweden & $\mathrm{T}$ & Plots without plants & $\begin{array}{l}\text { May-August } 1998 \\
\text { April-October } 1999\end{array}$ & 0.09 & 180 & 8 & 67 & 0.192 & 0.29 & Johansson et al. (2003) \\
\hline Domestic wastewater & Lakeus, Finland & B & P. australis, T. latifolia & $\begin{array}{l}\text { January 2002-October } \\
2003\end{array}$ & 44 & 3624 & 66.1 & 23 & 0.007 & 0.03 & Søvik et al. (2006) \\
\hline Domestic wastewater & Ruka, Finland & B & Carex-Sphagnum & January-October 2002 & 0.82 & 289 & 59.7 & 88 & 0.106 & 0.12 & Søvik et al. (2006) \\
\hline Domestic wastewater & $\begin{array}{l}\text { Skallstuggu } \\
\text { Norway }\end{array}$ & $\mathrm{T}$ & P. australis & $\begin{array}{l}\text { July 2001-February } \\
2002\end{array}$ & n.a. & n.a. & n.a. & 69.2 & 0.041 & 0.006 & Søvik et al. (2006) \\
\hline Domestic wastewater & $\begin{array}{l}\text { Skjonhaug, } \\
\text { Norway }\end{array}$ & $\mathrm{T}$ & $\begin{array}{l}\text { I. pseudacorus, T. latifolia, S. } \\
\text { lacustris }\end{array}$ & $\begin{array}{l}\text { July 2001-February } \\
2002\end{array}$ & 0.4 & 300 & 43.4 & 136 & 0.094 & 0.07 & Søvik et al. (2006) \\
\hline Domestic wastewater & $\begin{array}{l}\text { Magle, } \\
\text { Hässleholm, } \\
\text { Sweden }\end{array}$ & $\mathrm{T}$ & J. effusus, T. latifolia, P. australis & April-May 2005 & 20 & 13,000 & 75 & 203 & 0.230 & 0.11 & Ström et al. (2006) \\
\hline Domestic wastewater & Skjønhaug, & $\mathrm{T}$ & I. pseudacorus, T. latifolia, S. & October 2001-July & 0.4 & 250 & 38 & 99 & 0.130 & 0.13 & Søvik and Kløve (2007) \\
\hline Domestic wastewater & Ibaraki, Japan & $\mathrm{T} / \mathrm{W}$ & P. australis & $\begin{array}{l}\text { August 2004-May } \\
2006\end{array}$ & 0.0012 & 0.5 & 73.5 & 128 & 0.079 & 0.06 & Gui et al. (2007) \\
\hline Domestic wastewater & Ibaraki, Japan & $\mathrm{T} / \mathrm{W}$ & P. australis & June-August 2006 & 0.0012 & 0.5 & 100 & 174 & 0.2 & 0.12 & Liu et al. (2009) \\
\hline $\begin{array}{l}\text { Agricult. non-point } \\
\text { pollution }\end{array}$ & Mesocosms, USA & T & P. australis, Typha spp. & $\begin{array}{l}33 \text { days in summer } \\
1998\end{array}$ & n.a. & n.a. & n.a. & 73 & 0.16 & 0.22 & Xue et al. (1999) \\
\hline $\begin{array}{l}\text { Agricult, non-point } \\
\text { pollution }\end{array}$ & $\begin{array}{l}\text { Donaumoos, } \\
\text { Germany }\end{array}$ & $\mathrm{T}$ & $\begin{array}{l}\text { Basin 1, T. latifolia, } T \text {. } \\
\text { angustifolia }\end{array}$ & $\begin{array}{l}\text { September 1998-May } \\
1999\end{array}$ & 2.2 & 158 & 51 & 15.3 & 0.009 & 0.06 & Wild et al. (2001) \\
\hline $\begin{array}{l}\text { Agricult, non-point } \\
\text { pollution }\end{array}$ & $\begin{array}{l}\text { Donaumoos, } \\
\text { Germany }\end{array}$ & $\mathrm{T}$ & $\begin{array}{l}\text { Basin 2, T. latifolia, } T \text {. } \\
\text { angustifolia }\end{array}$ & $\begin{array}{l}\text { September 1998-May } \\
1999\end{array}$ & 2.6 & 158 & 23 & 5.8 & -0.003 & -0.05 & Wild et al. (2001) \\
\hline $\begin{array}{l}\text { Agricult. non-point } \\
\text { pollution }\end{array}$ & Hovi, Finland & B & $\begin{array}{l}\text { T. latifolia, S. sylvaticus, A. } \\
\text { plantago-aquatica, } P . \\
\text { arundinacea, F. ulmaria, I. } \\
\text { pseudacorus, J. conglomeratus }\end{array}$ & $\begin{array}{l}\text { January 2002-October } \\
2003\end{array}$ & 0.6 & 78.6 & 1.4 & 1 & 0.001 & 0.07 & Søvik et al. (2006) \\
\hline Dairy farm wastewater & $\begin{array}{l}\text { Truro Nova } \\
\text { Scotia, Canada }\end{array}$ & $\mathrm{T}$ & 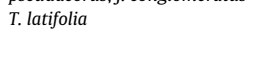 & $\begin{array}{l}\text { August } \\
2005-\text { September } \\
2006\end{array}$ & 0.00066 & 0.105 & 306 & 203 & 0.25 & 0.12 & Van der Zaag et al. (2010) \\
\hline Raw municipal wastewater & Jiaonan, China & $\mathrm{T}$ & $\begin{array}{l}\text { P. australis, A. calamus, L. } \\
\text { minor }\end{array}$ & $\begin{array}{l}\text { June 1999-August } \\
2000\end{array}$ & 49.5 & 12,000 & 120 & 121 & 0.068 & 0.06 & Tai et al. (2002) \\
\hline Synthetic wastewater & $\begin{array}{l}\text { Lab studies Jinan, } \\
\text { China }\end{array}$ & $\mathrm{W} / \mathrm{T}$ & P. australis & April-June 2008 & 0.000018 & 0.012 & 52 & 144 & 0.250 & 0.17 & Wu et al. (2009) \\
\hline Synthetic wastewater & $\begin{array}{l}\text { Lab studies Jinan, } \\
\text { China }\end{array}$ & $\mathrm{w} / \mathrm{T}$ & P. australis & April-June 2008 & 0.000018 & 0.012 & 55 & 153 & $0.650^{\mathrm{a}}$ & $0.43^{\mathrm{a}}$ & Wu et al. (2009) \\
\hline Synthetic wastewater & $\begin{array}{l}\text { Lab studies Jinan, } \\
\text { China }\end{array}$ & $\mathrm{w} / \mathrm{T}$ & P. australis & April-June 2008 & 0.000018 & 0.012 & 54 & 150 & 0.150 & 0.10 & Wu et al. (2009) \\
\hline Synthetic wastewater & $\begin{array}{l}\text { Lab studies Jinan. } \\
\text { China }\end{array}$ & $\mathrm{W} / \mathrm{T}$ & P. australis & April-June 2008 & 0.000018 & 0.012 & 51 & 142 & 0.1 & 0.07 & Wu et al. (2009) \\
\hline Synthetic wastewater & $\begin{array}{l}\text { Lab studies Jinan, } \\
\text { China }\end{array}$ & $\mathrm{W} / \mathrm{T}$ & P. australis & April-June 2008 & 0.000018 & 0.012 & 49 & 136 & 0.05 & 0.04 & Wu et al. (2009) \\
\hline
\end{tabular}

a Not included in the correlation analysis (see text). 
Table 3

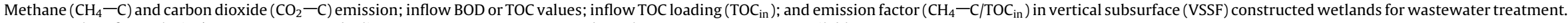

Average values for each site/event are presented. Climate zones: $\mathrm{T}$ - temperate, $\mathrm{B}$ - boreal, $\mathrm{W}$ - warm. n.a. - not available.

\begin{tabular}{|c|c|c|c|c|c|c|c|c|c|c|c|c|c|c|}
\hline Waste-water type & $\begin{array}{l}\text { Study site, } \\
\text { country }\end{array}$ & $\begin{array}{l}\text { Climate } \\
\text { zone }\end{array}$ & Plant species & Period of measurement & Area (ha) & $Q\left(\mathrm{~m}^{-3} \mathrm{~d}^{-1}\right)$ & $\begin{array}{l}\text { BOD } \\
\left(\mathrm{mg} \mathrm{L}^{-1}\right)\end{array}$ & $\begin{array}{l}\text { TOC } \\
\left(\mathrm{mgL}^{-1}\right)\end{array}$ & $\begin{array}{l}\text { Inflow BOD load } \\
\left(\mathrm{mg} \mathrm{m}^{-2} \mathrm{~h}^{-1}\right)\end{array}$ & $\begin{array}{l}\mathrm{TOC}_{\text {in }} \\
\left(\mathrm{mg} \mathrm{m}^{-2} \mathrm{~h}^{-1}\right)\end{array}$ & $\begin{array}{l}\mathrm{CO}_{2}-\mathrm{C} \text { emiss. } \\
\left(\mathrm{mg} \mathrm{m}^{-2} \mathrm{~h}^{-1}\right)\end{array}$ & $\begin{array}{l}\mathrm{CH}_{4}-\mathrm{C} \text { flux } \\
\left(\mathrm{mg} \mathrm{m}^{-2} \mathrm{~h}^{-1}\right)\end{array}$ & $\begin{array}{l}\mathrm{CH}_{4}-\mathrm{C} / \mathrm{TOC}_{\mathrm{in}} \\
(\%)\end{array}$ & References \\
\hline Domestic wastewater & Kõo, Estonia & $T / B$ & P. australis & October 2001-November 2003 & 0.0128 & 65 & 32.2 & 16.1 & 681 & 341 & 208 & 3 & 0.88 & $\begin{array}{l}\text { Teiter and Mander } \\
\text { (2005), Søvik et al. } \\
\text { (2006) and Mander et al. } \\
\text { (2008) }\end{array}$ \\
\hline Domestic wastewater & Ski, Norway & в & No vegetation & June-December 2001 & 0.000005 & 0.042 & n.a. & 40.5 & n.a. & 1418 & 127 & 5.4 & 0.38 & Søvik et al. (2006) \\
\hline Domestic wastewater & $\begin{array}{l}\text { Miho, Ibaraki } \\
\text { Japan }\end{array}$ & $\mathrm{T} / \mathrm{W}$ & P. australis & August 2004-May 2006 & 0.0012 & 0.5 & 107.3 & 10.3 & n.a. & 17.9 & n.a. & 0.30 & 1.68 & Gui et al. (2007) \\
\hline Domestic wastewater & $\begin{array}{l}\text { Miho, Ibaraki, } \\
\text { Japan }\end{array}$ & $\mathrm{T} / \mathrm{W}$ & P. australis & June-August 2006 & 0.0012 & 0.5 & 200 & n.a. & 347 & 174 & n.a. & 3 & 1.73 & Liu et al. (2009) \\
\hline
\end{tabular}

Table 4

Nitrous oxide $\left(\mathrm{N}_{2} \mathrm{O}-\mathrm{N}\right)$ emission; inflow total nitrogen loading $\left(\mathrm{TN}_{\text {in }}\right)$; and emission factor $\left(\mathrm{N}_{2} \mathrm{O}-\mathrm{N} / \mathrm{TN}_{\text {in }}\right)$ in vertical subsurface (VSSF) constructed wetlands for wastewater treatment. Average values for each site/event are presented. Climate zones: $\mathrm{T}$ - temperate, B - boreal, $\mathrm{W}$ - warm.

\begin{tabular}{|c|c|c|c|c|c|c|c|c|c|c|c|}
\hline Waste-water type & Study site, country & $\begin{array}{l}\text { Climate } \\
\text { zone }\end{array}$ & Plant species & Period of measurement & Area (ha) & $Q\left(m^{-3} d^{-1}\right)$ & $\mathrm{TN}\left(\mathrm{mg} \mathrm{L}^{-1}\right)$ & $\begin{array}{l}\mathrm{TN}_{\text {in }} \\
\left(\mathrm{mg} \mathrm{m}^{-2} \mathrm{~h}^{-1}\right)\end{array}$ & $\begin{array}{l}\mathrm{N}_{2} \mathrm{O}-\mathrm{N} \text { flux } \\
\left(\mathrm{mg} \mathrm{m}^{-2} \mathrm{~h}^{-1}\right)\end{array}$ & $\begin{array}{l}\mathrm{N}_{2} \mathrm{O}-\mathrm{N} / \mathrm{TN}_{\text {in }} \\
(\%)\end{array}$ & References \\
\hline Domestic wastewater & Kõo, Estonia & $T / B$ & P. australis & October 2001-November 2003 & 0.0128 & 65 & 50.9 & 1077 & 0.225 & 0.021 & $\begin{array}{l}\text { Teiter and Mander (2005) and } \\
\text { Søvik et al. (2006) }\end{array}$ \\
\hline Domestic wastewater & Ski, Norway & $\mathrm{T} / \mathrm{B}$ & No vegetation & June-December 2001 & 0.000005 & 0.0042 & 52.6 & 1841 & 0.200 & 0.011 & Søvik et al. (2006) \\
\hline Domestic wastewater & Miho, Ibaraki Japan & $\mathrm{T} / \mathrm{W}$ & P. australis & August 2004-May 2006 & 0.0012 & 0.5 & 73.5 & 128 & 0.123 & 0.096 & Gui et al. (2007) \\
\hline Domestic wastewater & Miho, Ibaraki, Japan & $\mathrm{T} / \mathrm{W}$ & P. australis & June-August 2006 & 0.0012 & 0.5 & 100 & 174 & 0.073 & 0.042 & Liu et al. (2009) \\
\hline Artificial wastewater & Ibaraki, Japan & $\mathrm{T} / \mathrm{W}$ & P. australis & April 2001-December 2002 & 0.000025 & 0.17 & 9.8 & 278 & 0.003 & 0.001 & Inamori et al. (2007) \\
\hline Artificial wastewater & Ibaraki, Japan & $\mathrm{T} / \mathrm{W}$ & P. australis & April 2001-December 2002 & 0.000025 & 0.17 & 18.4 & 521 & 0.008 & 0.001 & Inamori et al. (2007) \\
\hline Artificial wastewater & Ibaraki, Japan & $\mathrm{T} / \mathrm{W}$ & P. australis & April 2001-December 2002 & 0.000025 & 0.17 & 36.7 & 1040 & 0.033 & 0.003 & Inamori et al. (2007) \\
\hline Artificial wastewater & Ibaraki, Japan & $\mathrm{T} / \mathrm{W}$ & Zizania latifolia & April 2001-December 2002 & 0.000025 & 0.17 & 9.8 & 278 & 0.005 & 0.002 & Inamori et al. (2007) \\
\hline Artificial wastewater & Ibaraki, Japan & $\mathrm{T} / \mathrm{W}$ & Z. latifolia & April 2001-December 2002 & 0.000025 & 0.17 & 18.4 & 521 & 0.023 & 0.004 & Inamori et al. (2007) \\
\hline Artificial wastewater & Ibaraki, Japan & $\mathrm{T} / \mathrm{W}$ & Z. latifolia & April 2001-December 2002 & 0.000025 & 0.17 & 36.7 & 1040 & 0.040 & 0.004 & Inamori et al. (2007) \\
\hline Artificial wastewater & Ibaraki, Japan & $\mathrm{T} / \mathrm{W}$ & T. latifolia & January 2005-September 2006 & 0.000025 & 0.421 & 15 & 1053 & 0.200 & 0.019 & Inamori et al. (2008) \\
\hline Artificial wastewater & Ibaraki, Japan & $\mathrm{T} / \mathrm{W}$ & T. latifolia & January 2005-September 2006 & 0.000025 & 0.421 & 30 & 2105 & 0.353 & 0.017 & Inamori et al. (2008) \\
\hline Artificial wastewater & Ibaraki, Japan & $\mathrm{T} / \mathrm{W}$ & Z. latifolia & January 2005-September 2006 & 0.000025 & 0.421 & 15 & 1053 & 0.211 & 0.020 & Inamori et al. (2008) \\
\hline Artificial wastewater & Ibaraki, Japan & $\mathrm{T} / \mathrm{W}$ & Z. latifolia & January 2005-September 2006 & 0.000025 & 0.421 & 30 & 2105 & 0.424 & 0.020 & Inamori et al. (2008) \\
\hline Artificial wastewater & Ibaraki, Japan & $\mathrm{T} / \mathrm{W}$ & $\begin{array}{l}\text { P. australis } \\
\text { P. }\end{array}$ & January 2005-September 2006 & 0.000025 & 0.421 & 15 & 1053 & 0.188 & 0.018 & Inamori et al. (2008) \\
\hline Artificial wastewater & Ibaraki, Japan & $\mathrm{T} / \mathrm{W}$ & P. australis & January 2005-September 2006 & 0.000025 & 0.421 & 30 & 2105 & 0.371 & 0.018 & Inamori et al. (2008) \\
\hline Artificial wastewater & Ibaraki, Japan & $\mathrm{T} / \mathrm{W}$ & Non-vegetated control & January 2005-September 2006 & 0.000025 & 0.421 & 15 & 1053 & 0.150 & 0.014 & Inamori et al. (2008) \\
\hline Artificial wastewater & Ibaraki, Japan & $\mathrm{T} / \mathrm{W}$ & Non-vegetated control & January 2005-September 2006 & 0.000025 & 0.421 & 30 & 2105 & 0.162 & 0.008 & Inamori et al. (2008) \\
\hline Artificial wastewater & Ibaraki, Japan & $\mathrm{T} / \mathrm{W}$ & $\begin{array}{l}\text { P. australis, T. latifolia, Z. } \\
\text { latifolia }\end{array}$ & $\begin{array}{l}\text { January 2005-August } 2006 \\
\text {. }\end{array}$ & 0.000025 & 0.17 & 3.62 & 103 & 0.034 & 0.033 & Wang et al. (2008a,b) \\
\hline Artificial wastewater & Ibaraki, Japan & $\mathrm{T} / \mathrm{W}$ & P. australis, T. latifolia & January 2005-August 2006 & 0.000025 & 0.17 & 6.65 & 188 & 0.110 & 0.058 & Wang et al. (2008a,b) \\
\hline Artificial wastewater & Ibaraki, Japan & $\mathrm{T} / \mathrm{W}$ & P. australis, Z. Iatifolia & January 2005-August 2006 & 0.000025 & 0.17 & 4.5 & 128 & 0.043 & 0.034 & Wang et al. (2008a,b) \\
\hline
\end{tabular}


Table 5

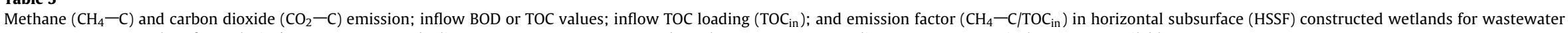
treatment. Average values for each site/event are presented. Climate zones: $\mathrm{T}$ - temperate, B - boreal, $\mathrm{W}$ - warm, M - Mediterranean, $\operatorname{Tr}$ - tropical. n.a. - not available.

\begin{tabular}{|c|c|c|c|c|c|c|c|c|c|c|c|c|c|c|}
\hline Waste-water type & $\begin{array}{l}\text { Study site, } \\
\text { country }\end{array}$ & $\begin{array}{l}\text { Climate } \\
\text { zone }\end{array}$ & Plant species & Period of measurement & Area (ha) & $Q\left(m^{-3} d^{-1}\right)$ & $\begin{array}{l}\text { BOD } \\
\left(\mathrm{mg} \mathrm{L}^{-1}\right)\end{array}$ & $\begin{array}{l}\mathrm{TOC} \\
\left(\mathrm{mg} \mathrm{L}^{-1}\right)\end{array}$ & $\begin{array}{l}\text { Inflow BOD load } \\
\left(\mathrm{mg} \mathrm{m}^{-2} \mathrm{~h}^{-1}\right)\end{array}$ & $\begin{array}{l}\mathrm{TOC}_{\text {in }} \\
\left(\mathrm{mg} \mathrm{m}^{-2} \mathrm{~h}^{-1}\right)\end{array}$ & $\begin{array}{l}\mathrm{CO}_{2}-\mathrm{C} \text { emission } \\
\left(\mathrm{mg} \mathrm{m}^{-2} \mathrm{~h}^{-1}\right)\end{array}$ & $\begin{array}{l}\mathrm{CH}_{4}-\mathrm{C} \text { flux } \\
\left(\mathrm{mg} \mathrm{m}^{-2} \mathrm{~h}^{-1}\right)\end{array}$ & $\begin{array}{l}\mathrm{CH}_{4}-\mathrm{C} / \mathrm{TOC}_{\mathrm{in}} \\
(\%)\end{array}$ & References \\
\hline $\begin{array}{l}\text { Domestic } \\
\text { wastewater }\end{array}$ & $\begin{array}{l}\text { Kodijärve, } \\
\text { Estonia }\end{array}$ & $T / B$ & $\begin{array}{l}\text { T. latifolia, } P \text {. } \\
\text { australis, Scirpus } \\
\text { sylvaticus }\end{array}$ & $\begin{array}{l}\text { October 2001-November } \\
2003\end{array}$ & 0.03125 & 2.85 & n.a. & 69 & n.a. & 26 & 99.2 & 2.6 & 9.9 & $\begin{array}{l}\text { Mander et al. (2003, } \\
\text { 2005a,b, 2008), Teiter and } \\
\text { Mander (2005), Søvik et al. } \\
\text { (2006) }\end{array}$ \\
\hline $\begin{array}{l}\text { Domestic } \\
\text { wastewater }\end{array}$ & Kõo, Estonia & $T / B$ & P. australis & $\begin{array}{l}\text { October 2001-November } \\
2003\end{array}$ & 0.0365 & 60 & 62.8 & n.a. & 430 & 215 & 41.7 & 3.09 & 1.4 & $\begin{array}{l}\text { Teiter and Mander (2005) } \\
\text { and Søvik et al. (2006) }\end{array}$ \\
\hline $\begin{array}{l}\text { Domestic } \\
\text { wastewater }\end{array}$ & Ski, Norway & B & No vegetation & June-December 2001 & 0.00009 & 0.072 & n.a. & 22.3 & n.a. & 74 & 51.9 & 7.1 & 9.6 & Søvik et al. (2006) \\
\hline $\begin{array}{l}\text { Domestic } \\
\text { wastewater }\end{array}$ & Barcelona, Spain & $\mathrm{T} / \mathrm{M}$ & $\begin{array}{l}\text { Non-vegetated } \\
\text { gravel from a CW }\end{array}$ & $\begin{array}{l}20 \text { days lab exper. in gravel } \\
\text { beds }\end{array}$ & 0.0055 & 1.98 & 200 & n.a. & 300 & 150 & 128 & 0.048 & 0.03 & Garcia et al. (2007) \\
\hline $\begin{array}{l}\text { Domestic } \\
\text { wastewater }\end{array}$ & $\begin{array}{l}\text { Miho, Ibaraki } \\
\text { Japan }\end{array}$ & $\mathrm{T} / \mathrm{W}$ & P. australis & August 2004-May 2006 & 0.0012 & 0.5 & 107.3 & 10.3 & n.a. & 17.9 & n.a., & 0.76 & 4.3 & Gui et al. (2007) \\
\hline $\begin{array}{l}\text { Domestic } \\
\text { wastewater }\end{array}$ & $\begin{array}{l}\text { Slavosovice } \\
\text { Czech Republic }\end{array}$ & $\mathrm{T}$ & P. australis & June-October 2004 & 0.0748 & 10.4 & n.a. & 38.1 & n.a. & 22.1 & 174.7 & $17.5^{\mathrm{a}}$ & $79.3^{\mathrm{a}}$ & Picek et al. (2007) \\
\hline $\begin{array}{l}\text { Domestic } \\
\text { wastewater }\end{array}$ & $\begin{array}{l}\text { Miho, Ibaraki, } \\
\text { Japan }\end{array}$ & $\mathrm{T} / \mathrm{W}$ & P. australis & June-August 2006 & 0.0012 & 0.5 & 200 & n.a. & 347 & 174 & n.a. & 7 & 4 & Liu et al. (2009) \\
\hline $\begin{array}{l}\text { Dairy farm } \\
\text { wastewater }\end{array}$ & $\begin{array}{l}\text { Truro, Nova } \\
\text { Scotia, Canada }\end{array}$ & $\mathrm{T}$ & T. latifolia & $\begin{array}{l}\text { August 2005-September } \\
2006\end{array}$ & 0.00066 & 0.105 & 186 & n.a. & 123.3 & 61.6 & 146 & 4.9 & 7.9 & Van der Zaag et al. (2010) \\
\hline Peat mining runoff & $\begin{array}{l}\text { Kompsasuo } \\
\text { Finland }\end{array}$ & B & $\begin{array}{l}\text { Sphagnum } \\
\text { angustifolium, S. } \\
\text { papillosum, } M . \\
\text { trifoliata, Carex } \\
\text { spp. }\end{array}$ & June-August 1992 & 1.9 & n.a. & n.a. & n.a. & n.a. & 16.9 & 303 & 5.8 & $34.3^{\mathrm{a}}$ & Liikanen et al. (2006) \\
\hline Peat mining runoff & $\begin{array}{l}\text { Kompsasuo } \\
\text { Finland }\end{array}$ & B & $\begin{array}{l}\text { M. trifoliata, C. } \\
\text { lasiocarpa, } P \text {. } \\
\text { palustris, Sphagn } \\
\text { spp. }\end{array}$ & August 2001-August 2002 & 1.9 & n.a. & n.a. & n.a. & n.a. & 15 & 567 & 16.7 & $111.3^{\mathrm{a}}$ & Liikanen et al. (2006) \\
\hline Peat mining runoff & $\begin{array}{l}\text { Kompsasuo, } \\
\text { Finland }\end{array}$ & B & $\begin{array}{l}\text { Sphagn spp., } \\
\text { Menyanthes } \\
\text { trifoliata, Carex } \\
\text { lasiocarpa, } \\
\text { Potentilla } \\
\text { palustris }\end{array}$ & $\begin{array}{l}\text { January 2002-October } \\
2003\end{array}$ & 2.4 & 1857 & n.a. & 15.0 & n.a. & 8.2 & n.a. & $13.1^{\mathrm{a}}$ & $160^{\mathrm{a}}$ & Søvik et al. (2006) \\
\hline $\begin{array}{l}\text { Landfill leachate } \\
\text { fresh }\end{array}$ & $\begin{array}{l}\text { Bangkok, } \\
\text { Thailand }\end{array}$ & $\operatorname{Tr}$ & T. angustifolia & January-December 2006 & 0.0003 & 0.056 & 5275 & 2816 & & $2190^{\mathrm{a}}$ & n.a. & 14.3 & 0.7 & Chiemchaisri et al. (2008) \\
\hline $\begin{array}{l}\text { Landfill leachate } \\
\text { stabilized }\end{array}$ & $\begin{array}{l}\text { Bangkok, } \\
\text { Thailand }\end{array}$ & $\operatorname{Trl}$ & T. angustifolia & January-December 2006 & 0.0003 & 0.056 & 42 & 403 & & 313 & n.a. & 9.25 & 3.0 & Chiemchaisri et al. (2008) \\
\hline
\end{tabular}


farm wastewater treatment in Friedelhausen, Germany (Fey et al., 1999) has also been included (Table 6).

Tanner et al. (1997) presented estimated values for inflow of total organic carbon (TOC in $_{\text {) }}$, Xue et al. (1999) for inflow total nitrogen $\left(\mathrm{TN}_{\mathrm{in}}\right)$, and Søvik et al. (2006) for both $\mathrm{TOC}_{\mathrm{in}}$ and $\mathrm{TN}_{\mathrm{in}}$. For most of the systems, $\mathrm{TOC}_{\text {in }}$ and $\mathrm{TN}_{\text {in }}$ values in $\mathrm{g} \mathrm{m}^{-2} \mathrm{~h}^{-1}$ were calculated based on area, hydraulic load and inflow TOC or BOD and TN concentration data.

The EF values were calculated as:

$\mathrm{EF}_{\mathrm{CH} 4}=\left(\mathrm{CH}_{4}-\right.$ Cemission $\left./ \mathrm{TOC}_{\mathrm{in}}\right) * 100(\%)$

$\mathrm{EF}_{\mathrm{N} 2 \mathrm{O}}=\left(\mathrm{N}_{2} \mathrm{O}-\mathrm{Nemission} / \mathrm{TN}_{\mathrm{in}}\right) * 100(\%)$

The $\mathrm{TOC}_{\text {in }}$ values were available for $8 \mathrm{FWS}$ CWs (Table 1 ), for all four VSSF CWs (Table 3), and for 6 HSSF CWs (Table 5). For the rest of the systems only biochemical oxygen demand (BOD) values were usable, and for these the following approximation based on domestic wastewater data was used: TOC $=0.5$ BOD (Garcia et al., 2007).

For the calculation of emission factors, we used data series from one year or at least a vegetation period. Some extreme values of $\mathrm{CH}_{4}$ emissions and related EF values from Jiaonan, Skjønhaug (Table 1), Kompsasuo and Slavosovice (Table 5), as well as one extreme value for $\mathrm{N}_{2} \mathrm{O}$ emission by Wu et al. (2009) (Table 2) were not used in further analyses. Likewise, we did not include in our study $\mathrm{CH}_{4}$ emission values from small-scale laboratory experiments by Inamori et al. (2007), Wang et al. (2008a,b), and Maltais-Landry et al. (2009a,b).

All of the GHG emission data in the analyzed publications were collected using the static chamber/gas-chromatograph technique (Hutchinson and Livingston, 1993). No publications could be found on the use of eddy covariance technique for measuring GHG fluxes in CWs.

\subsection{Statistical analysis of data}

The STATISTICA 7.1 program was used for the data analysis. We tested the normality of variables with Lilliefors' and Shapiro-Wilk's tests. All variables were log-transformed prior to data analysis. Differences in average $\mathrm{CH}_{4}$ and $\mathrm{N}_{2} \mathrm{O}$ emission values between wetland types and due to the presence of vegetation were tested using oneway analysis of variance (ANOVA). Stepwise multiple regression analysis with the forward selection option was applied to select predictor variables of $\mathrm{CH}_{4}$ and $\mathrm{N}_{2} \mathrm{O}$ emissions in the case of a particular wetland type. The level of significance $\alpha=0.05$ was accepted in all cases.

\section{Results}

\subsection{Carbon dioxide emission}

The emission of $\mathrm{CO}_{2}-\mathrm{C}$ was significantly lower in FWS CWs (ranging from 29.4 to 176.0, with average and median values of 92.3 and $95.8 \mathrm{mg} \mathrm{m}^{-2} \mathrm{~h}^{-1}$, respectively) than in subsurface flow CWs (VSSF+ HSSF; 51.9-567.0; average 184.7, median $137 \mathrm{mg} \mathrm{m}^{-2} \mathrm{~h}^{-1}$; Fig. 1A; Tables 1, 3 and 5).

There is a significant negative correlation between the inflow TOC loading $\left(\mathrm{TOC}_{\mathrm{in}}\right.$ ) value and $\mathrm{CO}_{2}-\mathrm{C}$ emission (Fig. $2 \mathrm{~A}$ ).

Likewise, we have found a positive correlation $(p<0.05)$ between the $\mathrm{CO}_{2}-\mathrm{C}$ emission and $\mathrm{CH}_{4}$ emission factor $\left(\mathrm{CH}_{4}-\mathrm{C} / \mathrm{TOC}_{\text {in }}\right.$ ) in all $\mathrm{CW}$ types (Fig. 2B), whereas this correlation was stronger $(p<0.01)$ in FWS CWs (Fig. 2 C).

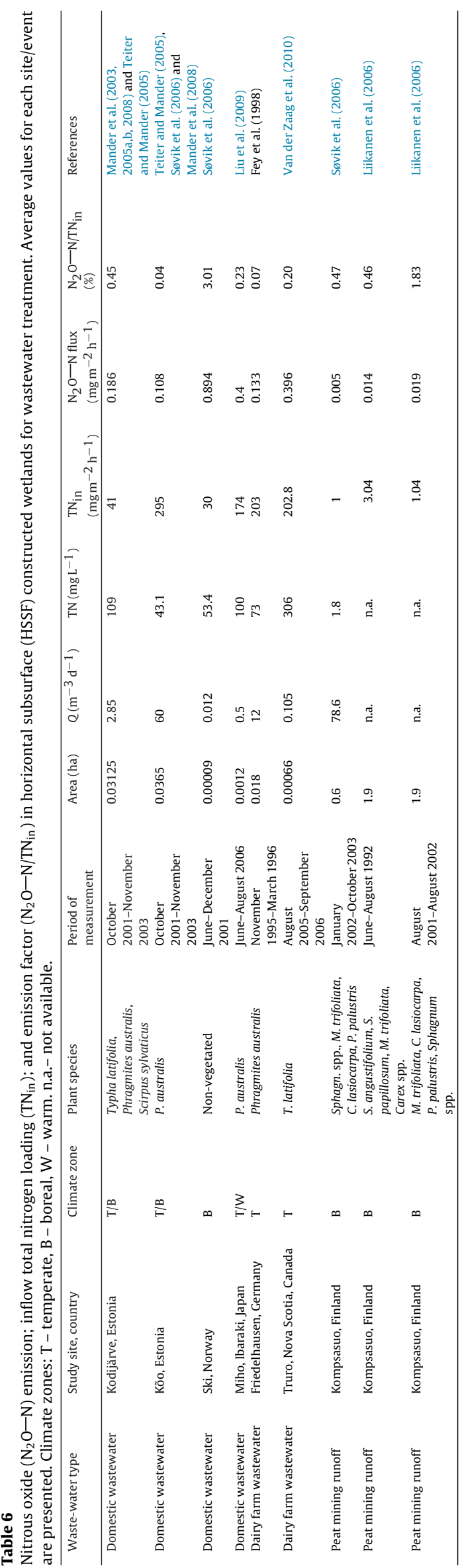




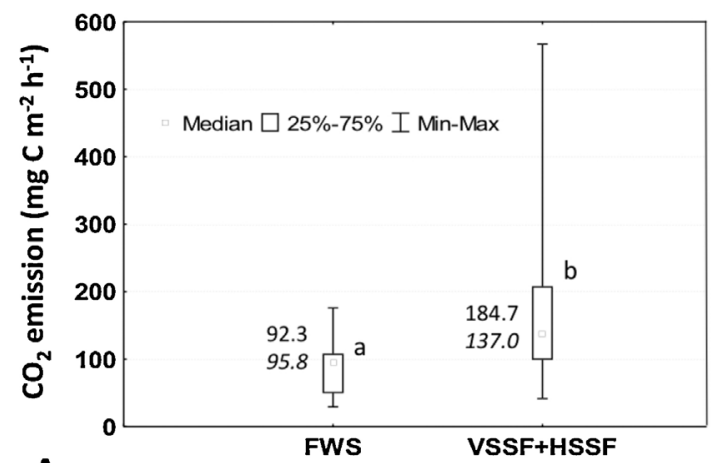

A
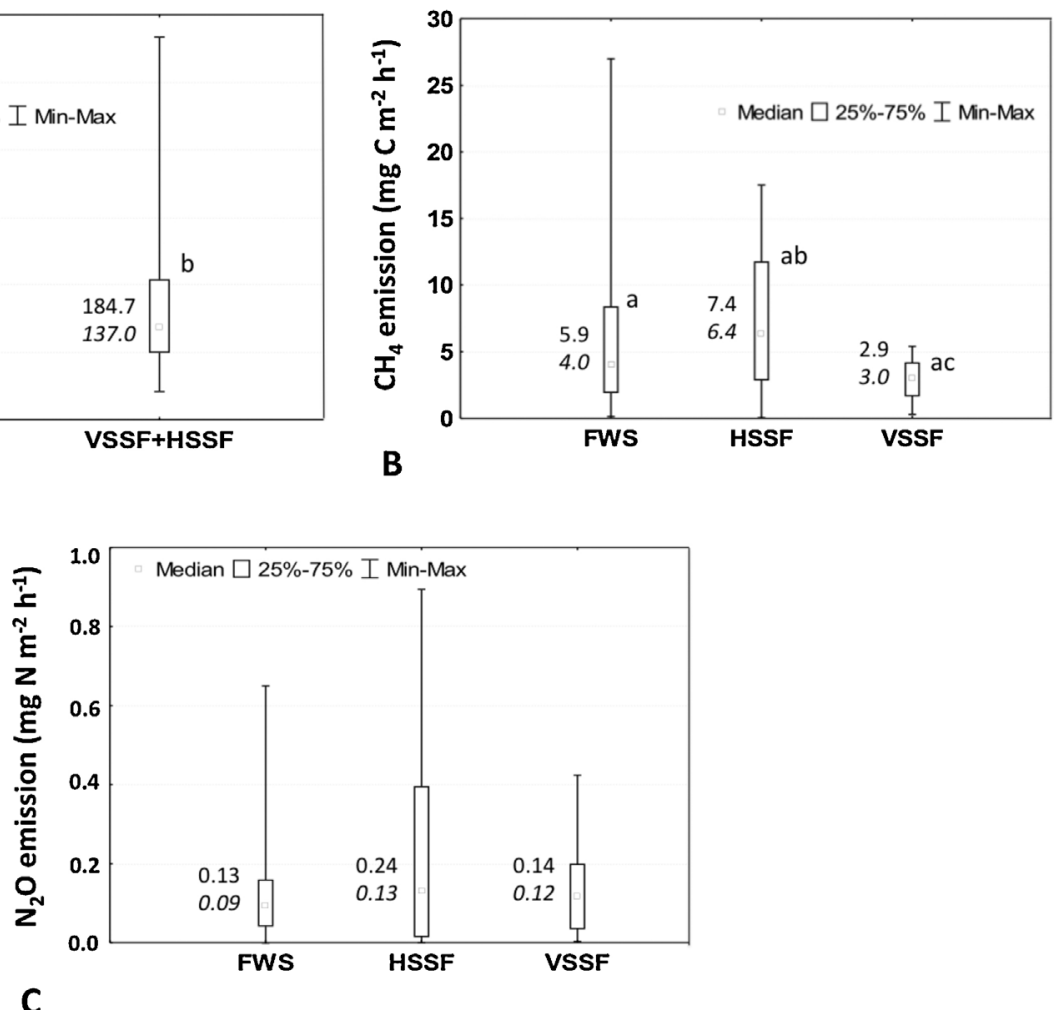

Fig. 1. Average, median, $25 \%$ and $75 \%$ quartile and $\min /$ max values of carbon dioxide (A), methane (B), and nitrous oxide (C) emissions in various types of constructed wetlands (CW). FWS - free water surface, VSSF - vertical subsurface flow, HSSF - horizontal subsurface flow CWs. Numbers close to boxes indicate average values (above) and median values (below, in italic). Different lower case letters indicate significant differences in emission values between various types of CWs ( $p<0.05$ ).
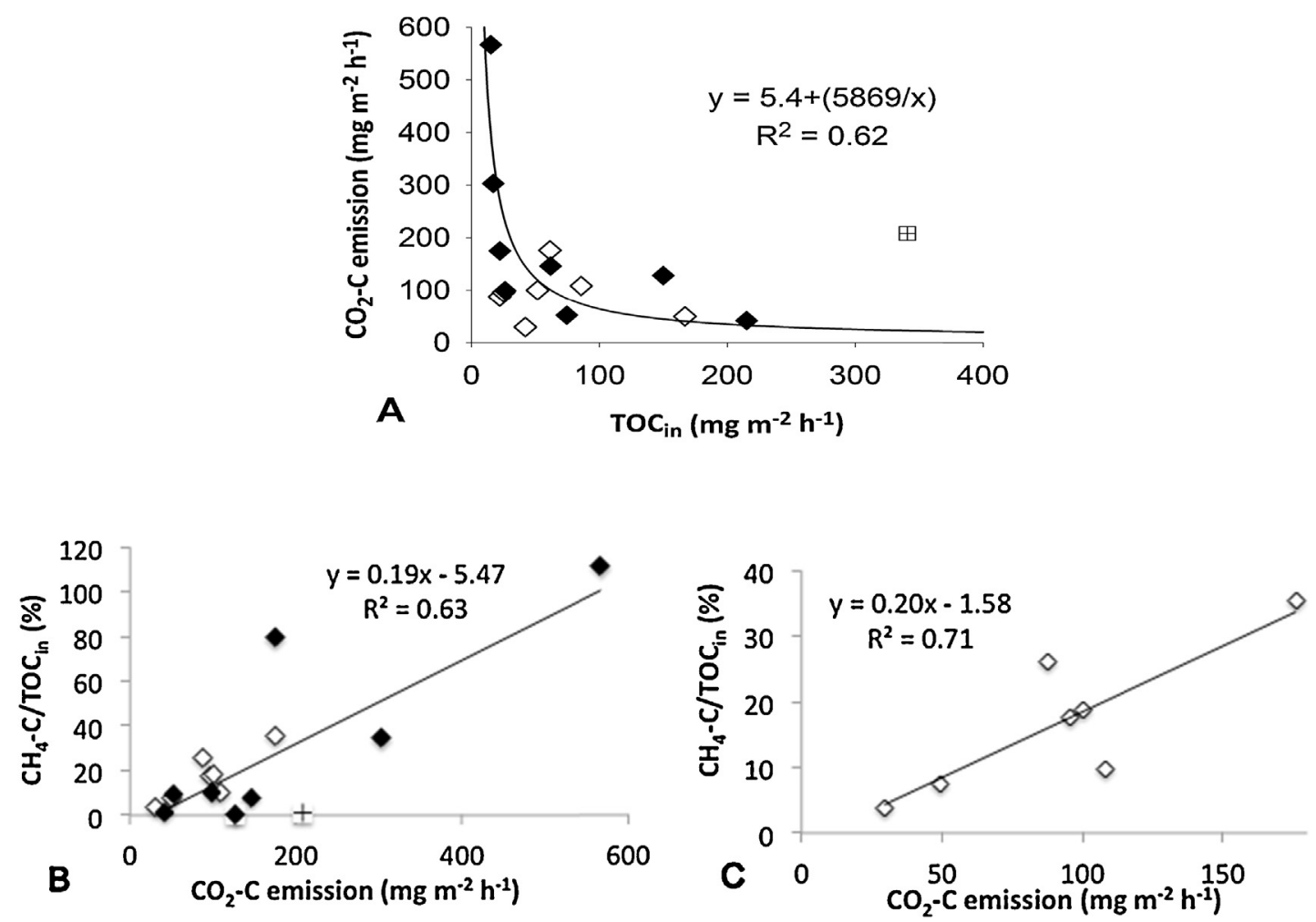

Fig. 2. The relationship between inflow TOC loading $\left(\mathrm{TOC}_{\mathrm{in}}\right)$ and $\mathrm{CO}_{2}-\mathrm{C}$ emission in all types of $\mathrm{CWs}$ studied $(\mathrm{A})$, and between the $\mathrm{CO}_{2}-\mathrm{C}_{2}$ emission and $\mathrm{CH}_{4}$ emission factor $\left(\mathrm{CH}_{4}-\mathrm{C} / \mathrm{TOC}_{\mathrm{in}}\right)$ for all types of CWs (B) and in FWS CWs (C). White diamonds - FWS, black diamonds - HSSF, and crosses - VSSF CWs. In A and B: $p<0.05$, in C: $p<0.01$. 
Table 7

Relationships between $\mathrm{CH}_{4}$ and $\mathrm{N}_{2} \mathrm{O}$ emission and wetland operational parameters $\left(\mathrm{TOC}_{\mathrm{in}}, \mathrm{TN}_{\mathrm{in}}, Q\right)$ based on stepwise multiple regression analysis.

\begin{tabular}{|c|c|c|c|c|c|}
\hline Dependent variable & Wetland type & Variables in model & Regression coefficient & $R^{2}$ value (\%) & $p$ Value \\
\hline $\mathrm{CH}_{4}$ emission & FWS & $\mathrm{TOC}_{\text {in }}$ & 0.58 & 30.0 & $<0.001$ \\
\hline $\mathrm{CH}_{4}$ emission & HSSF & $\mathrm{TOC}_{\mathrm{in}}$ & 0.34 & 40.4 & $<0.05$ \\
\hline $\mathrm{CH}_{4}$ emission & HSSF & $\mathrm{Q}$ & -0.31 & 40.4 & $<0.01$ \\
\hline $\mathrm{N}_{2} \mathrm{O}$ emission & FWS & $\mathrm{TN}_{\text {in }}$ & 0.02 & 23.7 & $<0.05$ \\
\hline $\mathrm{N}_{2} \mathrm{O}$ emission & VSSF & $\mathrm{TN}_{\mathrm{in}}$ & 0.07 & 43.9 & $<0.001$ \\
\hline $\mathrm{N}_{2} \mathrm{O}$ emission & HSSF & $\mathrm{TN}_{\mathrm{in}}$ & 0.06 & 23.9 & $<0.05$ \\
\hline
\end{tabular}

\subsection{Methane emission}

In VSSF CWs the $\mathrm{CH}_{4}-\mathrm{C}$ emission (range 0.3-5.4, average 2.9, median $3.0 \mathrm{mg} \mathrm{m}^{-2} \mathrm{~h}^{-1}$ ) was significantly lower than in HSSF CWs (0.048-17.5, 7.4 and $\left.6.4 \mathrm{mg} \mathrm{m}^{-2} \mathrm{~h}^{-1}\right)$ and FWS CWs $(0.15-27,5.9$ and $4.0 \mathrm{mg} \mathrm{m}^{-2} \mathrm{~h}^{-1}$ ) (Fig. 1B). Multiple regression analysis yielded a significant correlation between the $\mathrm{TOC}_{\mathrm{in}}$ and $\mathrm{CH}_{4}-\mathrm{C}$ emission values in all types of CWs (Table 7 and Fig. 3).

Significant differences have been found between the EF values in different CW types. The highest value was found for FWS CWs, in which on average $16.9 \%$ of inflow TOC is transformed into $\mathrm{CH}_{4}-\mathrm{C}$ (median value being 18.0; Table 8). This is followed by HSSF CWs, where the average and median EF values are $4.5 \%$ and $3.8 \%$, respectively, whereas in VSSF CWs only $1.17 \%$ (median value $1.28 \%$ ) of $\mathrm{TOC}_{\text {in }}$ is transformed to methane (Table 8 ).

\subsection{Nitrous oxide emission}

There were no significant differences in $\mathrm{N}_{2} \mathrm{O}-\mathrm{N}$ emissions in various CW types (Table 8). The lowest values were found in FWS CWs (ranging from -0.003 to 0.65 ; average 0.13 , median $\left.0.09 \mathrm{mg} \mathrm{m}^{-2} \mathrm{~h}^{-1}\right)$, followed by VSSF CWs (0.003-0.424; average 0.14 , median $0.12 \mathrm{mg} \mathrm{m}^{-2} \mathrm{~h}^{-1}$ ) and HSSF CWs (0-0.894, average 0.24 , median $0.13 \mathrm{mg} \mathrm{m}^{-2} \mathrm{~h}^{-1}$ ) (Fig. 1C). All of the CW types showed a significant positive correlation between the inflow TN loading and $\mathrm{N}_{2} \mathrm{O}-\mathrm{N}$ emission values (Table 8 and Fig. 4).
Average $\mathrm{N}_{2} \mathrm{O}$ emission factors differed significantly in all three CW types: $0.79 \%$ (median $0.34 \%$ ) in HSSF, $0.13 \%$ (median $0.11 \%$ ) in FWS, and $0.023 \%$ (median $0.018 \%$ ) in VSSF CWs (Table 9).

\section{Discussion}

\subsection{Carbon dioxide emission}

Although $\mathrm{CO}_{2}$ is a major GHG, there are only a limited number of studies reporting on $\mathrm{CO}_{2}$ emissions in CWs (Mander et al., 2003a,b, 2005a,b, 2008; Teiter and Mander, 2005; Liikanen et al., 2006; Garcia et al., 2007; Søvik et al., 2006; Van der Zaag et al., 2010). The values of $\mathrm{CO}_{2}$ emissions (soil + root + rhizome respiration + litter decomposition) vary from 0.88 to $2.37 \mathrm{~kg} \mathrm{CO}_{2}-\mathrm{Cha}^{-1} \mathrm{yr}^{-1}$ (Garcia et al., 2007) in anaerobic HSSF CWs, up to $3840-7360 \mathrm{~kg} \mathrm{CO}_{2}-\mathrm{Cha}^{-1} \mathrm{yr}^{-1}$ in a well-aerated HSSF CW with a lowered water table and well-developed macrophyte cover (Scirpus sylvaticus, Phragmites australis) (Mander et al., 2008). Some of the emitted $\mathrm{CO}_{2}$ will be assimilated by plants, and in optimal conditions the carbon sequestration in the HSSF CW filter material can reach 15,500-20,750 $\mathrm{kg} \mathrm{CO}_{2}-\mathrm{Cha}^{-1} \mathrm{yr}^{-1}$ (Mander et al., 2008). This carbon is, however, non-stable and can be easily mineralized when the water table lowers or when the filter material, which becomes saturated with phosphorus, is replaced with a new one.
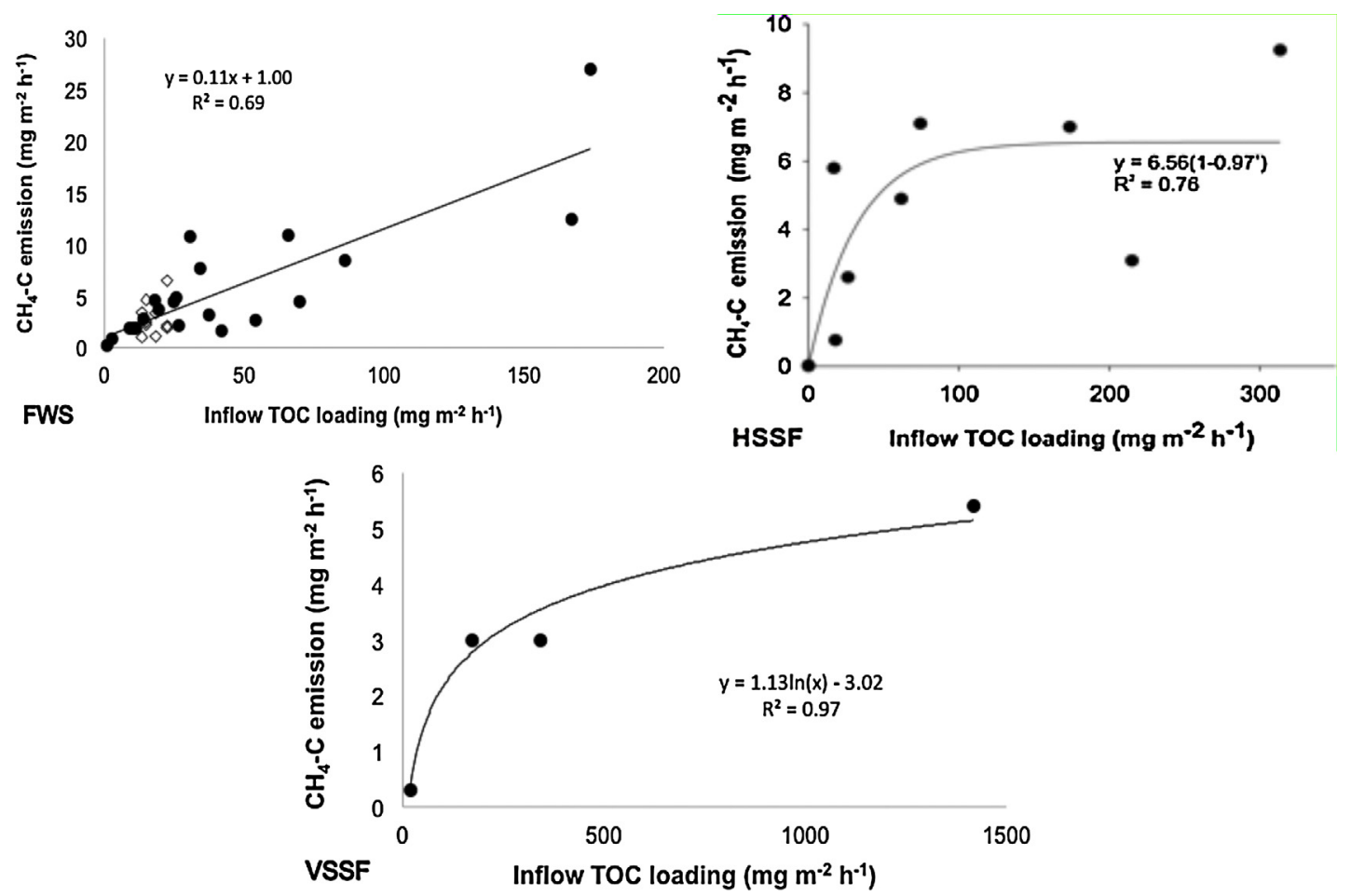

Fig. 3. The relationship between inflow TOC loading $\left(\mathrm{TOC}_{\text {in }}\right)$ and $\mathrm{CH}_{4}-\mathrm{C}$ emission in FWS, VSSF and HSSF CWs $(\mathrm{p}<0.05)$. 
Table 8

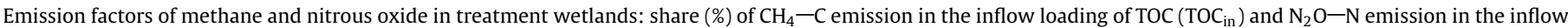
loading of $\mathrm{TN}\left(\mathrm{TN}_{\mathrm{in}}\right)$. The letters a-c and $\mathrm{x}-\mathrm{z}$ indicate significant $(p<0.05)$ differences between $\mathrm{CH}_{4}$ and $\mathrm{N}_{2} \mathrm{O}$ emission factors, respectively.

\begin{tabular}{|c|c|c|c|c|c|c|c|c|}
\hline \multirow[t]{2}{*}{ Type of wetland } & \multicolumn{4}{|c|}{$\mathrm{CH}_{4}-\mathrm{C} / \mathrm{TOC}_{\mathrm{in}}(\%)$} & \multicolumn{4}{|c|}{$\mathrm{N}_{2} \mathrm{O}-\mathrm{N} / \mathrm{TN}_{\text {in }}(\%)$} \\
\hline & Average & Median & Standard Error & Number of analyses & Average & Median & Standard Error & Number of analyses \\
\hline Free water surface (FWS) wetlands & $16.9^{\mathrm{a}}$ & 18.0 & 1.8 & 22 & $0.13^{x}$ & 0.11 & 0.024 & 24 \\
\hline Horizontal subsurface flow (HSSF) wetlands & $4.5^{\mathrm{b}}$ & 3.8 & 1.1 & 9 & $0.79^{y}$ & 0.34 & 0.38 & 8 \\
\hline Vertical subsurface flow (VSSF) wetlands & $1.17^{\mathrm{c}}$ & 1.28 & 0.33 & 4 & $0.023^{z}$ & 0.018 & 0.005 & 22 \\
\hline
\end{tabular}
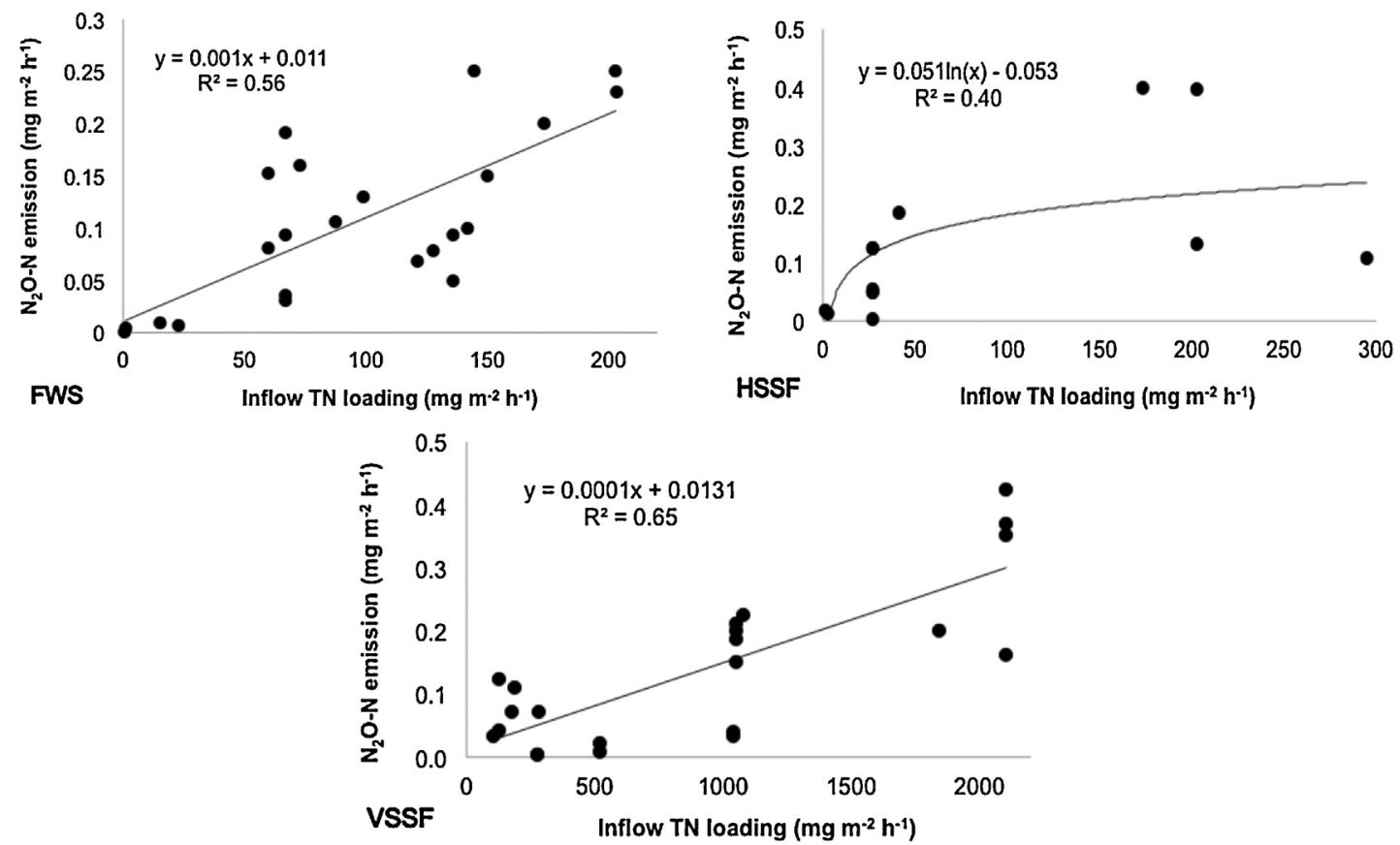

Fig. 4. The relationship between inflow $\mathrm{TN}$ loading $\left(\mathrm{TN}_{\mathrm{in}}\right)$ and $\mathrm{N}_{2} \mathrm{O}-\mathrm{N}$ emission in FWS, VSSF, and $\mathrm{HSSF}$ CWs $(\mathrm{p}<0.05)$.

Table 9

Selected factors and processes influencing $\mathrm{CH}_{4}$, and $\mathrm{N}_{2} \mathrm{O}$ emission in constructed wetlands.

\begin{tabular}{|c|c|c|}
\hline Influencing factors/processes & $\mathrm{CH}_{4}$ emission & $\mathrm{N}_{2} \mathrm{O}$ emission \\
\hline Higher water/soil/air temperature & Increase in almost all cases ${ }^{a, b, c, d, e, f}$ with few exceptions ${ }^{g}$ & No clear relationship a,b,c,d,g,h \\
\hline Higher moisture in soil or filter material (higher value of WFPS) & Clear increase $^{i, j}$ & Decrease $^{i, j}$ \\
\hline Higher wastewater loading & Increase $^{\mathrm{a}, \mathrm{b}, \mathrm{c}, \mathrm{d}, \mathrm{k}, \mathrm{l}, \mathrm{m}}$ & Increase $\mathrm{a}^{\mathrm{a}, \mathrm{b}, \mathrm{d}, \mathrm{n}}$ \\
\hline Presence of aerenchymal plants & Increase $^{0, p, q} /$ decrease (depends on conditions) $)^{r}$ & Increase $^{\mathrm{s}} /$ decrease $^{\mathrm{q}, \mathrm{t}}$ \\
\hline Pulsing hydrological regime (intermittent loading) & Clear decrease $\mathrm{e}^{\mathrm{i}, \mathrm{u}}$ & Increase $\mathrm{e}^{\mathrm{i}, \mathrm{v}, \mathrm{w}} /$ decrease in some SF CWs $\mathrm{s}^{\mathrm{x}}$ \\
\hline Deeper water table (from surface) in HSSF CWs & Decrease $^{i, j}$ & Increase $^{i, j}$ \\
\hline
\end{tabular}

a Mander et al. (2003).

b Mander et al. (2005a,b).

c Teiter and Mander (2005).

d Søvik et al. (2006).

e Kayranli et al. (2010).

f Van der Zaag et al. (2010).

g Søvik and Kløve (2007).

h Fey et al. (1999).

i Mander et al. (2011).

j Yang et al. (2013).

k Tanner et al. (1997).

1 Tai et al. (2002).

m Picek et al. (2007).

n Hunt et al. (2009).

o Inamori et al. (2007).

p Inamori et al. (2008).

q Wang et al. (2008a,b).

$\mathrm{r}$ Maltais-Landry et al. (2009a,b).

s Rückauf et al. (2004).

t Silvan et al. (2005).

u Altor and Mitsch (2006).

v Jia et al. (2011).

w Van de Riet et al. (2013).

x Hernandez and Mitsch (2006). 
Our analysis showed that $\mathrm{CO}_{2}$ emission from CWs is negatively correlated with $\mathrm{TOC}_{\text {in }}$ in HSSF CWs (Fig. 2A). Above a certain $\mathrm{TOC}_{\text {in }}$ level $\left(>100 \mathrm{mg} \mathrm{C} \mathrm{m}^{-2} \mathrm{~h}^{-1}\right)$ the treatment wetlands do not more than $200 \mathrm{mg} \mathrm{CO}_{2}-\mathrm{C} \mathrm{m}^{-2} \mathrm{~h}^{-1}$, which indicate that above that $\mathrm{TOC}_{\text {in }}$ value the purification process also no longer intensifies. Likewise, $\mathrm{CH} 4$ emission is stabilized above the $\mathrm{TOC}_{\text {in }}$ value $>100 \mathrm{mg} \mathrm{C} \mathrm{m}^{-2} \mathrm{~h}^{-1}$ (Fig. 3).

The $\mathrm{CO}_{2}$ emission factor $\left(\mathrm{CO}_{2}-\mathrm{C} / \mathrm{TOC}_{\mathrm{in}}\right)$ varied from $9 \%$ to $3780 \%$, and was in most cases higher than $100 \%$. The smallest $\mathrm{CO}_{2} \mathrm{EF}$ values were measured in VSSFs and HSSFs with scarce plant cover (Tables 1, 3 and 5). This clearly shows the significant role of aquatic plants, which bring a great deal of atmospheric $\mathrm{CO}_{2}$ into the system, thereby establishing the basis for $\mathrm{C}$ sequestration (Mitsch and Gosselink, 2007).

Eddy covariance data were not available in CWs, although the studies conducted in an abandoned agricultural peat meadow, which can be considered to be a proxy ecosystem to HSSF CWs, showed a significant variation in net ecosystem exchange (NEE), namely -446 and $-232 \mathrm{~g} \mathrm{C} \mathrm{m}^{-2} \mathrm{yr}^{-1}$ in wet and dry years, respectively. $\mathrm{CO}_{2}$ emission has decreased significantly as result of the raised water table, while $\mathrm{CH}_{4}$ fluxes have increased. Over the whole study period (2004-2006), the area was a small net GHG sink, given as $\mathrm{CO}_{2}$-eq. of $-86 \mathrm{~g} \mathrm{C} \mathrm{m}^{-2} \mathrm{yr}^{-1}$ (Hendriks et al., 2007).

Nevertheless, with the right design takes into consideration all of the regulation and supporting services of CW systems, it would be possible to achieve significant $C$ sequestration such as that which takes place in natural wetlands (Mitsch and Gosselink, 2007; Mitsch et al., 2013).

\subsection{Methane emission}

Methane seems to be the most important GHG emitted from CWs. In FWS CWs an average of $16.9 \%$ of $\mathrm{TOC}_{\text {in }}$ is transformed to $\mathrm{CH}_{4}-\mathrm{C}$ (Table 8). This is due to the intensive accumulation of organic matter (OM) in CWs (Mander et al., 2014). Some of the formed methane is probably oxidised and emitted as $\mathrm{CO}_{2}$, which is supported by a significant correlation of $\mathrm{CO}_{2}-\mathrm{C}$ emission and $\mathrm{CH}_{4}$ EF value $\left(\mathrm{CH}_{4}-\mathrm{C} / \mathrm{TOC}\right.$; Fig. $2 \mathrm{~B}$ and $\left.\mathrm{C}\right)$. The non-linear character of $\mathrm{CH}_{4}-\mathrm{C}$ emission vs. the $\mathrm{TOC}_{\text {in }}$ relationship in HSSF and VSSF CWs (Fig. 3) may indicate some limit level for $\mathrm{CH}_{4}$ release, i.e., from a certain $\mathrm{TOC}_{\text {in }}$ loading, methanogenesis may be inhibited by increased concentration of ammonia, or accumulation of volatile fatty acids inhibiting the rate limiting step of methanogenesis, hydrolysis of organic matter (Mata-Alvarez et al., 2000). However, the limited amount of data available prevented us from drawing more comprehensive conclusions. On the other hand, excessively heavy loading leads to the clogging of the system, requiring corresponding management activity. Also, very high overloading, such as in the case of FWS CWs, rarely happens in practice.

Overloaded FWS CWs such as the Jiaonan wetland system for raw wastewater treatment (Tai et al., 2002; Table 1) and the Skjønhaug wetland (Søvik and Kløve, 2007; Table 1) with a high OM accumulation rate had EF values that even exceeded 100\%. Likewise, the Slavosovice HSSF CW (Picek et al., 2007; Table 5) showed very high $\mathrm{EF}$ values (79\%), which is probably due to overloading and clogging problems in the system. The Kompsasuo peat mining wastewater treatment system in a Finnish peatland is a mixture of HSSF and FWS CWs (Liikanen et al., 2006), and is therefore considered under both categories (Søvik et al., 2006) or as an FWS CW (Mander et al., 2013). In this paper we follow the approach proposed by Liikanen et al. (2006) and consider the Kompsasuo treatment system as an HSSF CW (Table 5). This system does, however, also generate high values of $\mathrm{CH}_{4}$ emission, whereas during the period from 1992 to 2002, EF increased from 34.3\% to 111.3\% (Liikanen et al., 2006; Table 5). For the period 2002-2003, calculations based on the data of Søvik et al. (2006) give an EF value as high as $160 \%$ (Table 5 ). The data from all of the overloaded systems described above, as well as from the peat extraction wastewater treatment systems, were not included in our further analysis.

\subsection{Nitrous oxide emission}

Findings from earlier studies on GHG emissions in HSSF and VSSF CWs which show high $\mathrm{N}_{2}$ and low $\mathrm{N}_{2} \mathrm{O}$ emission - indicators of almost complete denitrification - suggest denitrification might be the main source of nitrous oxide (Mander et al., 2003, 2005a, 2008; Teiter and Mander, 2005). Heterotrophic denitrification relies on the absence of $\mathrm{O}_{2}$ for the conversion of $\mathrm{N}_{2} \mathrm{O}$ to $\mathrm{N}_{2}$; if oxygen is introduced during this last step, incomplete denitrification will occur and $\mathrm{N}_{2} \mathrm{O}$ will be released as the end product instead of $\mathrm{N}_{2}$. In wetland systems, where both ammonia and nitrate are present, the intermittent loading in VSSF CWs (Jia et al., 2011) and the fluctuating water table in HSSF CWs (Mander et al., 2011) which bring intermittent oxygen to the system can result in increased emissions by affecting both nitrification (through nitrifier stress, thus triggering nitrifier denitrification) and denitrification (by interrupting the last biochemical step; Dotro et al., 2011). The introduction of artificial aeration in laboratory-scale HSSF CWs (Maltais-Landry et al., 2009a,b) resulted in increased emissions; it is, however, unclear if this was due to greater air stripping of the dissolved gas or as a result of increased nitrification rates. In a laboratory-scale VSSF CW, Zhou et al. (2008) found a significant positive correlation between the $\mathrm{N}_{2} \mathrm{O}-\mathrm{N}$ flux and redox potential, suggesting nitrification was the dominant $\mathrm{N}_{2} \mathrm{O}$-emitting microbial process. Yu et al. (2006) found that redox potential was a significant indicator of $\mathrm{N}_{2} \mathrm{O}$ emission in soils of Gulf coast forests: maximum $\mathrm{N}_{2} \mathrm{O}$ concentration in these soils was found at about $\mathrm{Eh}+250 \mathrm{mV}$. However, additional measurement of $\mathrm{N}_{2}$ emissions and isotope analysis are needed to confirm these statements.

The character of $\mathrm{N}_{2} \mathrm{O}-\mathrm{N}$ emission vs. the $\mathrm{TN}_{\text {in }}$ relationship (Fig. 4) may also support the denitrification hypothesis: in contrast to the linear correlation in FWS and VSSF CWs, the relation is non-linear in HSSF CWs, in which dominating anaerobic conditions support complete denitrification. Higher than $50 \mathrm{mg} \mathrm{N} \mathrm{m}^{-2} \mathrm{hr}^{-1}$ of $\mathrm{TN}_{\text {in }}$ loading (Fig. 4) may enhance the anaerobic conditions in HSSFs, and if there is enough carbon in the system (which is a common case in HSSFs) the denitrification might be completed and the main product will be $\mathrm{N}_{2}$.

Likewise, studies on the filling modes of a sequencing batch reactor (SBR) demonstrated that a major emission of $\mathrm{N}_{2} \mathrm{O}$ took place at the aerobic phase, while $\mathrm{N}_{2} \mathrm{O}$ emission at the anoxic phase was insignificant (Park et al., 2001).

\subsection{Impact of physical, hydrological and operational factors}

Table 9 presents a brief overview of selected factors and processes that control $\mathrm{CH}_{4}$ and $\mathrm{N}_{2} \mathrm{O}$ emission in CWs. Higher inflow loading of both TOC and TN always increases the respective $\mathrm{CH}_{4}$ and $\mathrm{N}_{2} \mathrm{O}$ fluxes. This is also demonstrated by the significant correlation between the inflow TOC and TN values and the corresponding $\mathrm{CH}_{4}$ and $\mathrm{N}_{2} \mathrm{O}$ fluxes (Figs. 3 and 4). In most cases, the higher value of water-filled pore space (WFPS) of soils/sediments or the higher moisture of the filter material increased $\mathrm{CH}_{4}$ emission and decreased $\mathrm{N}_{2} \mathrm{O}$ emission. Likewise, the deeper water table (from surface) in $\mathrm{HSSF}$ CWs always decreased $\mathrm{CH}_{4}$ emission and increased $\mathrm{N}_{2} \mathrm{O}$ emission (Table 9). In arable soils, however, the highest $\mathrm{N}_{2} \mathrm{O}$ emission was found at WFPS values between $50 \%$ and $80 \%$ (Vilain et al., 2010). With few exceptions (Søvik and Kløve, 2007), the higher temperature of the environment always increased $\mathrm{CH}_{4}$ 
emission, whereas in terms of $\mathrm{N}_{2} \mathrm{O}$ the relationship is unclear. Our analysis along all $\mathrm{CW}$ types did not yield a significant relationship with $\mathrm{CH}_{4}$ and $\mathrm{N}_{2} \mathrm{O}$ emission and climate zone (see Tables 1-6), although there was a slight increasing trend in $\mathrm{CH}_{4}$ emission toward boreal CWs.

Wu et al. (2009) demonstrate that in FWS CWs the higher inflow COD concentration causes a significant increase in $\mathrm{N}_{2} \mathrm{O}$ emission, although our review analysis was unable to find a significant correlation between $\mathrm{TOC}_{\mathrm{in}}$ and $\mathrm{N}_{2} \mathrm{O}$ emission.

The location of sampling sites in the flow path is an additional important factor for GHG emission. In most cases, the $\mathrm{CH}_{4}$ emission in the inflow part of FWS and HSSF CWs has been significantly higher than in the outflow part (Tanner et al., 1997; Mander et al., 2005a,b; Søvik et al., 2006; Picek et al., 2007); some investigations have noted the same effect regarding the $\mathrm{N}_{2} \mathrm{O}$ flux (Mander et al. (2003) for an HSSF CW; Hernandez and Mitsch (2006) and Pulou (2011) for artificial riverine wetlands), and some for both $\mathrm{CH}_{4}$ and $\mathrm{N}_{2} \mathrm{O}$ emission (Mander et al., 2005a,b; Teiter and Mander, 2005; Søvik et al., 2006).

The ratio of soil/sediment $\mathrm{C} / \mathrm{N}$ is an important ecosystem parameter controlling many processes (Kalbitz et al., 2000). Yan et al. (2012) determined that the optimum C/N ratio is 5:1, at which point VSSF CWs can achieve relatively high biological nutrient removal efficiency and a low level of $\mathrm{CO}_{2}$ and $\mathrm{CH}_{4}$ emission. A low $\mathrm{C} / \mathrm{N}$ ratio may, however, significantly increase $\mathrm{N}_{2} \mathrm{O}$ emission. This has been shown to occur in forested histosols (Klemedtsson et al., 2005) and activated sludge plants for domestic wastewater treatment, where $20-30 \%$ of the influent $\mathrm{N}$ was converted to $\mathrm{N}_{2} \mathrm{O}$ at a C/N ratio below 3.5 (Itokawa et al., 2001). Van der Zaag et al. (2010) report the same phenomenon for FWS and HSSF CWs: $\mathrm{N}_{2} \mathrm{O}$ emissions increased when influent wastewater had a low $\mathrm{C} / \mathrm{N}$ ratio. This shift toward increased $\mathrm{N}_{2} \mathrm{O}$ emission at lower $\mathrm{C} / \mathrm{N}$ ratio values may be due to alterations in the structure of the microbial community involved in nitrogen transformation in $\mathrm{CW}$, and particularly in denitrifying microbial species. A low $\mathrm{C} / \mathrm{N}$ ratio may favor microbial species with incomplete denitrification pathways (lack of nitrous oxide reductase encoding nosZ gene), which leads to an increase in $\mathrm{N}_{2} \mathrm{O}$ as an end product of denitrification.

It is known that iron and sulphur compounds significantly suppress $\mathrm{CH}_{4}$ emission in peatlands (Dise et al., 1993). Decreases in $\mathrm{CH}_{4}$ emissions measured after the addition of $\mathrm{Fe}^{3+}$ to paddy soils (Jäckel and Schnell, 2000; Huang et al., 2009) and $\mathrm{SO}_{2}{ }^{-4}$ to rice field soils (Van der Gon and Neue, 1994) and histosols (Dise and Verry, 2001; Gauci et al., 2002) have been attributed to the inhibition of methanogenesis, predominantly through the stimulation of $\mathrm{SO}_{2}{ }^{-4}$ and $\mathrm{Fe}^{3+}$ reducing bacteria which out-compete methanogens for acetate and hydrogen. Stimulation of $\mathrm{CH}_{4}$ oxidation by $\mathrm{SO}_{2}{ }^{-4}$ (under anaerobic and aerobic conditions) and $\mathrm{Fe}^{3+}$ addition (under anaerobic conditions) may also contribute to these observed decreases in $\mathrm{CH}_{4}$ emissions (Kumaraswamy et al., 2001). On the other hand, in laboratory conditions $\mathrm{Fe}^{3+}$ application to paddy soil increased $\mathrm{N}_{2} \mathrm{O}$ emission (Huang et al., 2009).

However, there are only few examples on the effect of $\mathrm{Fe}^{3+}$ and $\mathrm{SO}_{4}{ }^{2-}$ on $\mathrm{CH}_{4}$ emission in CWs. For instance, the application of 10 tons of gypsum $\left(\mathrm{CaSO}_{4} \cdot 2 \mathrm{H}_{2} \mathrm{O}\right)$ per hectare to the sediment core of a FWS CW treating farm wastewater in Scotland reduced $\mathrm{CH}_{4}$ emissions by $28 \%$, whereas the addition of 5 tons of ochre $\left(\mathrm{Fe}(\mathrm{OH})_{3}\right.$ and $\mathrm{FeO}(\mathrm{OH})) \mathrm{ha}^{-1}$ caused methane emission to decrease by $63 \%$ (Pangala et al., 2009).

\subsection{The role of hydrological regime and plants}

The impact of hydrological regime (pulsing hydrology) and aquatic macrophytes seems to be more problematic, and different studies sometimes yield contradictory results (Table 9).
In all cases, however, the pulsing hydrological regime in FWS CWs and sometimes HSSF CWs, and intermittent loading in VSSF CWs clearly decrease methane emission. Regarding $\mathrm{N}_{2} \mathrm{O}$ emission, some studies (Jia et al., 2011; Mander et al., 2011) show enhanced $\mathrm{N}_{2} \mathrm{O}$ release from surface flow CWs due to pulsing hydrology. A similar effect was shown by Van de Riet et al. (2013) for rewetted peatlands. Some other studies on artificial riverine wetlands, however, suggest that pulsing hydrology slightly decreases $\mathrm{N}_{2} \mathrm{O}$ emission (Hernandez and Mitsch, 2006).

Based on stepwise multiple regression analysis, we found a significant negative correlation between the hydraulic load $(\mathrm{Q}$; Table 7) and $\mathrm{CH}_{4}$ emission in HSSF CWS, however, suggesting an increase in the hydraulic load would decrease the water purification efficiency of the system, and the benefit of lower $\mathrm{CH}_{4}$ release would be diminished.

Several studies show that extensive aquatic macrophyte cover significantly suppresses $\mathrm{CH}_{4}$ emission in FWS CWs (Stadmark and Leonardson, 2005; Thiere et al., 2011; Mander et al., 2013) and artificial riverine wetlands (Altor and Mitsch, 2006; Sha et al., 2011). Hernandez and Mitsch (2006, 2007), Pulou (2011), and Thiere et al. (2011) have also shown the decreasing effect of aquatic macrophyte cover on $\mathrm{N}_{2} \mathrm{O}$ emission from FWS wetlands. However, considering all of the aquatic macrophytes individually, the effect on $\mathrm{CH}_{4}$ and $\mathrm{N}_{2} \mathrm{O}$ emission can vary from decreasing to increasing. Some aerenchymatous macrophytes such as common reed $(P$. australis), which facilitate gas transport from the sediment $\left(\mathrm{CH}_{4}\right)$ and to the sediment $\left(\mathrm{O}_{2}\right)$, can inhibit $\mathrm{CH}_{4}$ emission from wetlands (Brix, 1990, 1997; Ström et al., 2005). Likewise, oxygenation and the related methanogenesis-inhibiting effect have been demonstrated in an experimental landfill-leachate-treatment wetland with willow plants (Williams et al., 2010). On the other hand, several aerenchymatous wetland plants such as Eriophorum vaginatum (Waddington et al., 1996; Ström et al., 2005), Juncus effusus (Smialek et al., 2006; Schafer et al., 2012), Typha latifolia and Zizania latifolia (Inamori et al., 2007; Wang et al., 2008a,b) are important emitters of methane. Also, in some conditions $P$. australis can emit $\mathrm{CH}_{4}$ (Juutinen et al., 2003; Duan et al., 2006).

Different wetland macrophytes show a remarkable variety in the results of $\mathrm{N}_{2} \mathrm{O}$ emission. Due to $\mathrm{N}$ uptake, the $\mathrm{N}_{2} \mathrm{O}$ emission in plant-covered CWs can be decreased (Silvan et al., 2005; Wang et al., 2008a,b; Maltais-Landry et al., 2009a,b). In contrast, Rückauf et al. (2004) showed in lab experiments that Phragmites plants significantly enhanced $\mathrm{N}_{2} \mathrm{O}$ emission, whereas Phalaris arundinacea did not affect $\mathrm{N}_{2} \mathrm{O}$ emissions, and no emission via the shoots was observed. Most probably, Phragmites plants were supplying more oxygen into the soil than the Phalaris.

The harvesting of Phragmites stands had a short-term decreasing effect on $\mathrm{CH}_{4}$ emission from FWS CWs, but harvesting in HSSF CWs did not impact GHG emissions (Zhu et al., 2007). Nevertheless, following the multiple ecosystem services concept (Mitsch and Gosselink, 2007), FWS CWs can be used for both water quality improvement and plant biomass production that does not generate GHG emissions. However, further long-term studies in the field of the macrophytes' effect and their possible use for multiple purposes are needed (see Meerburg et al., 2010).

The relationship between algae growth and GHG emissions in treatment wetlands has not been thoroughly studied. Our study shows that in FWS CWs for domestic wastewater treatment, beds with philamentous algae of Spirogyra spp. emitted more $\mathrm{N}_{2} \mathrm{O}$ and less $\mathrm{CH}_{4}$ than beds with aquatic macrophytes and non-vegetated beds (Tables 1 and 2). Anderson and Mitsch (2006) have found that in created riverine wetlands in deeper open water (OW) zones with sometimes intensive algae growth, mean sediment accumulation was significantly higher than that in zones of emergent vegetation. Large accumulations of $\mathrm{Ca}$ and inorganic $\mathrm{C}$ in the $\mathrm{OW}$ zones of 
wetlands suggest that $\mathrm{CaCO}_{3}$ deposition has remained a critical process where algae productivity has been highest (Anderson and Mitsch, 2006). These data suggest that algae growth may be a significant facilitator of $\mathrm{C}$ sequestration in FWS CWs, although in the earlier stages of FWS CWs this can, however, can lead an increase in BOD in the outlet (Diaz et al., 2012).

Constructed wetlands accumulate OM over time (Mander et al., 2008, 2014; Van der Zaag et al., 2010), forming in FWS CWs thicker organic layers and causing oxygen deficiency, which facilitates methanogenesis and results in elevated $\mathrm{CH}_{4}$ emission. To mitigate the negative role in the earlier stages after wetland creation, support of aquatic macrophytes' cover and its controlled management is recommended in the further application of FWS and HSSF CWs.

\subsection{Significance of emission values found}

The emission factors reported here are limited to the process emissions and therefore do not include the amount of GHG produced during the generation of electric power which is necessary, for example, for feeding batch wetlands though the use of pumps (particularly VSSF), artificial aeration in aerated wetlands, or for running activated sludge processes. The total operational carbon footprint of a treatment technology will be a combination of the process emissions (i.e., emission factors reported here) and the operational carbon associated with energy use, estimated at $0.544 \mathrm{~kg} \mathrm{CO}_{2}$ per $\mathrm{kWh}$ used (UKWIR, 2008). The amount of energy required to run a particular treatment plant is dependent on many factors, including the type of treatment technology (i.e., biofilm vs. suspended growth), topography (i.e., gravity vs. pumped flows), efficiency of the instrumentation (i.e., size of the blower or pump), instrument run times (i.e., how many batch cycles are operated), among many others. Thus, analogous to the conventional energy benchmarking among treatment plants to determine process efficiency in terms of energy used (and its associated carbon footprint) and proposing optimization strategies, it is also possible to compare treatment efficiency in terms of mass of GHG emitted as process emissions generated from removing pollutants from wastewater (i.e., emission factors).

In comparison with conventional wastewater treatment systems, CWs emit significantly lower levels of $\mathrm{N}_{2} \mathrm{O}$. Czepiel et al. (1995) show that the total $\mathrm{N}_{2} \mathrm{O}$ emissions from the municipal wastewater treatment systems in Durham, NH, USA were estimated to be $6.6 \times 10^{7} \mathrm{~g}$ of $\mathrm{N}_{2} \mathrm{O} \mathrm{yr}^{-1}$ from primary treatment and $1.2 \times 10^{9} \mathrm{~g}$ of $\mathrm{N}_{2} \mathrm{Oyr}^{-1}$ from secondary activated sludge treatment. The hourly rates of $\mathrm{N}_{2} \mathrm{O}$ emission ranged from 5 to $75 \mathrm{mg} \mathrm{N}_{2} \mathrm{O}-\mathrm{N} \mathrm{m}^{-2} \mathrm{~h}^{-1}$ over all parts of the system (Czepiel et al., 1995), which is one to three magnitudes higher than the emission from wastewater treatment wetlands (Tables 2, 4 and 6). Likewise, Park et al. (2000) measured significantly higher $\mathrm{N}_{2} \mathrm{O}$ emission values in a domestic wastewater treatment SBR than those found in CWs.

However, comparisons of CW emissions with energy intensive wastewater treatment systems (e.g., activated sludge plants) are possible by examining the emission factors and comparing those against systems with similar treatment goals. That is, comparison of hourly emissions per $\mathrm{m}^{2}$ is not the best way, as the volume of the treatment systems (i.e., $4-5 \mathrm{~m}$ aeration tanks vs. $0.6-1 \mathrm{~m}$ deep wetlands) and the area requirement per person equivalent are far too different for direct comparisons $\left(0.05-0.1 \mathrm{~m}^{2} \mathrm{PE}^{-1}\right.$ for activated sludge vs. $3-5 \mathrm{~m}^{2} \mathrm{PE}^{-1}$ for CWs). In this context, the EF of VSSF systems should typically be compared against the EF of other nitrifying systems, and FWS and HSSF systems should be compared against denitrifying or TN removing systems.

The mean $\mathrm{EF}$ of $0.023 \%$ of $\mathrm{TN}_{\text {in }}$ from nitrifying VSSF wetlands is significantly lower than the $0.16-0.6 \%$ reported for biological nutrient removal plants (Ahn et al., 2010), and the $0.036 \%$ from a nitrifying activated sludge plant (Aboobakar et al., 2013).

For HSSF wetlands, the mean emission factor of $0.79 \%$ of influent $\mathrm{TN}$ is within the range of $0.01-1.8 \%$ reported for energy intensive technologies (Ahn et al., 2010).

The life cycle assessment (LCA) in combination with GHG emission values shows the benefits of CWs. Pan et al. (2011) demonstrate that a conventional wastewater treatment plant system in China emits $7.3 \mathrm{~kg} \mathrm{CO}$-equivalents $\left(\mathrm{CO}_{2}\right.$-eq. including both $\mathrm{CO}_{2}, \mathrm{CH}_{4}$, and $\mathrm{N}_{2} \mathrm{O}$ fluxes calculated based on radiative force coefficients; IPCC, 2007) to remove $1 \mathrm{~kg}$ BOD in the studied life cycle, while the VSSF CW system only emits $3.18 \mathrm{~kg} \mathrm{CO}_{2}$-eq. To a large extent, this is due to significantly lower $\mathrm{CH}_{4}$ emission in the studied VSSF CWs. Although the EF value of $\mathrm{CH}_{4}$ emissions in FWS CWs is high (Table 8) the final efficiency of these systems may even be higher due to the lower need for construction materials and energy than in VSSF CWs (Vymazal, 2001, 2007). Similarly, the LCA of subsurface flow CWs, which included GHG emission, showed that in comparison with HSSF CWs, the VSSF CWs are less impactful configuration for wastewater treatment, especially for removing TN from domestic wastewater (Fuchs et al., 2011).

However, the EF values for $\mathrm{N}_{2} \mathrm{O}$ in all analyzed $\mathrm{CW}$ types and even in studied conventional wastewater treatment systems are lower than those found on agricultural soils in Great Britain, where annual EFs varied from $0.4 \%$ to $6.5 \%$ of the $\mathrm{N}$ applied (Dobbie and Smith, 2003), and were comparable with the present IPCC default $\mathrm{EF}$ for $\mathrm{N}_{2} \mathrm{O}$ of $1.25 \%$ of the $\mathrm{N}$ applied (IPCC, 2006). Thus, the mitigation of GHG emissions from treatment systems should be tackled alongside mitigation strategies for emissions from agricultural activities.

\subsection{Further study perspectives}

Among further investigations that would contribute to a better understanding of $\mathrm{C}$ and $\mathrm{N}$ cycling, the continuous measurement of $\mathrm{CO}_{2}, \mathrm{CH}_{4}$ and $\mathrm{N}_{2} \mathrm{O}$ fluxes using transparent automatic chamber method and eddy covariance technology can be highlighted. For VSSF and HSSF CWs with relatively small areas, the chamber method gives adequate estimates, although automatic and transparent chambers make it possible to fill the measurement gaps and analyze full $\mathrm{C}$ and $\mathrm{N}$ budgets (see, e.g., Chojnicki et al., 2010). In larger FWS CWs the eddy covariance method is preferable.

In order to distinguish between different sources of $\mathrm{N}_{2} \mathrm{O}$ (denitrification vs. nitrification), $\mathrm{N}_{2} \mathrm{O}$ isotopomer studies (Meijide et al., 2010; Well et al., 2012; Wunderlin et al., 2013) have yielded promising results and must be used in further investigations in wetlands. Some studies (Riya et al., 2010) suggest distinguishing between the direct and indirect GHG emissions in CWs, similarly to analogous studies on $\mathrm{N}_{2} \mathrm{O}$ fluxes from agricultural landscapes (IPCC, 2007; Vilain et al., 2012) and global estimations of $\mathrm{N}_{2} \mathrm{O}$ emissions (Seitzinger and Kroeze, 1998). Riya et al. (2010) evaluated direct $\mathrm{CH}_{4}$ and $\mathrm{N}_{2} \mathrm{O}$ emissions (gas fluxes from the $\mathrm{CW}$ surface water to the atmosphere) and indirect $\mathrm{CH}_{4}$ and $\mathrm{N}_{2} \mathrm{O}$ emissions (dissolved gas concentrations in filtered water) from a VSSF CW planted with forage rice, and found that the percentages of indirect emission to total (direct + indirect) emission during the experimental period were $2.9 \%$ and $86.7 \%$ for $\mathrm{CH}_{4}-\mathrm{C}$ and $\mathrm{N}_{2} \mathrm{O}-\mathrm{N}$, respectively. Emission factor of the indirect $\mathrm{N}_{2} \mathrm{O}-\mathrm{N}$ emission was $0.053-0.86 \%$. This is comparable to those of indirect emission sources in the literature and also to the results of our study. However, these results indicate the importance of monitoring and controlling indirect $\mathrm{N}_{2} \mathrm{O}-\mathrm{N}$ emission from CWs (see also Well et al., 2005, 2012). In terms of better performance and optimal maintenance of CWs, studies on the impact of hydrological regimes and the seasonal development 
of vegetation and microbial communities on the emission of GHGs and on $\mathrm{C}$ and $\mathrm{N}$ budgets must be carried out.

Better understanding of the role of microorganisms in key processes influencing $\mathrm{CH}_{4}$ and $\mathrm{N}_{2} \mathrm{O}$ emissions, methanogenesis and denitrification is another important challenge for further investigations. Analysis of the structure of microbial communities and functional gene abundance and diversity studies using next generation sequencing and quantitative PCR techniques are already widely used, and the first results for FWS CWs (Garcia-Lledo et al., 2011) and artificial riverine wetlands (Song et al., 2012; Ligi et al., 2014a,b) have been published.

\section{Conclusions}

The emission factor of $\mathrm{CH}_{4}$ in FWS and HSSF CWs has been found to be very high, yet the absolute value of $\mathrm{CH}_{4}$ emission is relatively small, and 1-2 magnitudes lower than that found in conventional wastewater treatment plants. Nevertheless, GHG emission is an important factor in the operation of CWs, but the main task is the optimization of water purification processes.

The use of peatlands in the creation of treatment wetlands has benefits regarding water quality improvement, but can significantly increase $\mathrm{CH}_{4}$ emission. The overloading of HSSF CWs will lead to clogging and to elevated $\mathrm{CH}_{4}$ emission, and the use of FWS CWs for raw wastewater treatment will also cause intensive OM accumulation but very high $\mathrm{CH}_{4}$ emission.

To mitigate $\mathrm{CH}_{4}$ emission from FWS and HSSF CWs, a pulsing hydrological regime (fluctuating water table and intermittent loading) is recommended. This can, however, elevate $\mathrm{N}_{2} \mathrm{O}$ emission, since the global warming potential (GWP) of these systems is mainly determined by $\mathrm{CH}_{4}$ emissions, and the intermittent loading and pulsing hydrology can be practical. Likewise, support for the development of aquatic macrophyte cover and controlled harvesting in FWS and HSSF CWs can help minimize GHG emissions.

\section{Acknowledgements}

This study was supported by the Estonian Research Council (grant IUT2-16); and the EU through the European Regional Development Fund (Center of Excellence ENVIRON).

\section{References}

Aboobakar, A., Cartmell, E., Stephenson, T., Jones, M., Vale, P., Dotro, G., 2013. Nitrous oxide emissions and dissolved oxygen profiling in a full-scale nitrifying activated sludge treatment plant. Water Res. 47 (2), 524-534

Ahn, J.H., Kim, S., Park, H., Rahm, B., Pagilla, K., Chandran, K., 2010. $\mathrm{N}_{2} \mathrm{O}$ emissions from activated sludge processes, 2008-2009: results of a national monitoring survey in the United States. Environ. Sci. Technol. 44 (12), 4505-4511.

Altor, A.E., Mitsch, W.J., 2006. Methane flux from created riparian marshes: relationship to intermittent versus continuous inundation and emergent macrophytes. Ecol. Eng. 28 (3), 224-234.

Anderson, C.J., Mitsch, W.J., 2006. Sediment, carbon, and nutrient accumulation at two 10-year-old created riverine marshes. Wetlands 26 (3), 779-792.

Arp, D.J., Stein, L.Y., 2003. Metabolism of inorganic N compounds by ammonia oxidizing bacteria. Crit. Rev. Biochem. Mol. 38, 471-495.

Bachand, P.A.M., Horne, A.J., 2000a. Denitrification in constructed free-water surface wetlands: I. Very high nitrate removal rates in a macrocosm study. Ecol. Eng. 14 (1/2), 9-15.

Bachand, P.A.M., Horne, A.J., 2000b. Denitrification in constructed free-water surface wetlands: II. Effects of vegetation and temperature. Ecol. Eng. 14 (1/2), 17-32.

Batson, J.A., Mander, Ü., Mitsch, W.J., 2012. Denitrification and a nitrogen budget of created riparian wetlands. J. Environ. Qual. 41, 2024-2032.

Brix, H., 1987. Treatment of wastewater in the rhizosphere of wetland plants - the root zone method. Water Sci. Technol. 19 (10), 107-118.

Brix, H., 1990. Gas exchange through the soil-atmosphere interface and through dead culms of Phragmites australis in a constructed wetland receiving domestic sewage. Water Res. 24, 377-389.

Brix, H., 1997. Do macrophytes play a role in constructed treatment wetlands? Water Sci. Technol. 35 (5), 11-17.
Chiemchaisri, C., Chiemchaisri, W., Junsod, J., Threedeach, S., Wicranarachchi, P.N., 2008. Leachate treatment and greenhouse gas emission in subsurface horizontal flow constructed wetland. Bioresour. Technol. 100 (16), 3808-3814.

Chojnicki, B.H., Michalak, M., Acosta, M., Juszczak, R., Augustin, J., Drösler, M., Olejnik, J., 2010. Measurements of carbon dioxide fluxes by chamber method at the Rzecin Wetland Ecosystem, Poland. Pol. J. Environ. Stud. 19 (2), 283-291.

Colliver, T., Stephenson, T., 2000. Production of nitrogen oxide and dinitrogen oxide by autotrophic nitrifiers. Biotechnol. Adv. 18 (3), 219-232.

Cooper, P.F., 2005. The performance of vertical flow constructed wetland systems with special reference to the significance of oxygen transfer and hydraulic loading rates. Water Sci. Technol. 51 (9), 81-90.

Cooper, P.F., Job, G.D., Green, M.B., Shutes, R.B.E., 1996. Reed Beds and Constructed Wetlands for Wastewater Treatment. WRc Publications, Medmenham, Marlow, UK.

Czepiel, P., Crill, P., Harriss, R., 1995. Nitrous oxide emissions from municipal wastewater treatment. Environ. Sci. Technol. 29, 2352-2366.

Diaz, F.J., O'Geen, A.T., Dahlgren, R.A., 2012. Agricultural pollutant removal by constructed wetlands: implications for water management and design. Agric. Water Manage. 104, 171-183.

Dise, N.B., Verry, E.S., 2001. Suppression of peatland methane emission by cumulative sulfate deposition in simulated acid rain. Biogeochemistry 53, 143-160.

Dise, N.B., Gorham, E., Verry, E.S., 1993. Environmental factors controlling methane emissions from peatlands in northern Minnesota. J. Geophys. Res. 98, 10583-10594

Dobbie, K.E., Smith, K.A., 2003. Nitrous oxide emission factors for agricultural soils in Great Britain: the impact of soil water-filled pore space and other controlling variables. Global Change Biol. 9 (2), 204-218.

Dotro, G., Jefferson, B., Jones, M., Cartmell, E., Vale, P., Stephenson, T., 2011. A review of the impact and potential of intermittent aeration on continuous flow nitrifying activated sludge. Environ. Technol. 32 (15), 1-13.

Duan, X., Wang, X., Ouyang, Z., 2006. Plant-mediated $\mathrm{CH}_{4}$ emission from a Phragmites-dominated wetland in an arid region, China. J. Freshwater Ecol. 21 (1), 1349-2145

Fey, A., Benckiser, G., Ottow, J.C.G., 1999. Emissions of nitrous oxide from a constructed wetland using a groundfilter and macrophytes in waste-water purification of a dairy farm. Biol. Fertil. Soils 29 (4), 354-359.

Fuchs., V.J., Mihelcic, J.R., Gierke, J.S., 2011. Life cycle assessment of vertical and horizontal flow constructed wetlands for wastewater treatment considering nitrogen and carbon greenhouse gas emissions. Water Res. 45 (5), 2073-2081.

Garcia, J., Capel, V., Castro, A., Ruiz, I., Soto, M., 2007. Anaerobic biodegradation tests and gas emissions from subsurface flow constructed wetlands. Bioresour. Technol. 98 (16), 3044-3052.

Garcia-Lledo, A., Vilar-Sanz, A., Trias, R., Hallin, S., Baneras, L., 2011. Genetic potential for $\mathrm{N}_{2} \mathrm{O}$ emissions from the sediment of a free water surface constructed wetland. Water Res. 45 (17), 5621-5632.

Gauci, V., Dise, N., Fowler, D., 2002. Controls on suppression of methane flux from a peat bog subjected to simulated acid rain sulfate deposition. Global Biogeochem. Cycle 16 (1), http://dx.doi.org/10.1029/200GB001370.

Goreau, T.J., Kaplan, W.A., Wofsky, S.C., McElroy, M.B., Valois, F.W., Watson, S.W., 1980. Production of $\mathrm{NO}_{2}{ }^{-}$and $\mathrm{N}_{2} \mathrm{O}$ by nitrifying bacteria at reduced concentrations of oxygen. Appl. Environ. Microbiol. 40, 526-532.

Gui, P., Inamori, R., Matsumura, M., Inamori, Y., 2007. Evaluation of constructed wetlands by waste water purification ability and greenhouse gas emissions. Water Sci. Technol. 56 (3), 49-55.

Hendriks, D.M.D., van Huissteden, J., Dolman, A.J., van der Molen, M.K., 2007. The full Greenhouase gas balance of an abandoned meadow. Biogeosciences 4 (3), 411-424.

Hernandez, M.E., Mitsch, W.J., 2006. Influence of hydrologic pulses, flooding frequency, and vegetation on nitrous oxide emissions from created riparian marshes. Wetlands 26, 862-877.

Hernandez, M.E., Mitsch, W.J., 2007. Denitrification in created riverine wetlands: influence of hydrology and season. Ecol. Eng. 30 (1), 78-88.

Huang, B., Yu, K.W., Robert, P.G., 2009. Effects of ferric iron reduction and regeneration on nitrous oxide and methane emissions in a rice soil. Chemosphere 74 , $481-486$

Huang, L., Gao, X., Guo, J., Ma, X., Liu, M., 2013. A review on the mechanism and affecting factors of nitrous oxide emission in constructed wetlands. Environ. Earth Sci. 68, 2171-2180.

Hunt, P.G., Stone, K.C., Matheny, T.A., Poach, M.E., Vanotti, M.B., Ducey, T.F., 2009. Denitrification of nitrified and non-nitrified swine lagoon wastewater in the suspended sludge layer of treatment wetlands. Ecol. Eng. 35 (10), 1514-1522

Hutchinson, G.L., Livingston, G.P., 1993. Use of chamber systems to measure trace gas fluxes. In: Harper, L.A., Mosier, A.R., Duxbury, J.M., Rolston, D.E. (Eds.), Agricultural Ecosystems Effects on Trace Gases and Global Climate Change. ASA Spec. Publ. 55. Am. Soc. Agron., Madison, WI, pp. 1-55.

Inamori, R., Gui, P., Dass, P., Matsumura, M., Xu, K.-Q., Kondo, T., Ebie, Y., Inamori, Y., 2007. Investigating $\mathrm{CH}_{4}$ and $\mathrm{N}_{2} \mathrm{O}$ emissions from eco-engineering wastewater treatment processes using constructed wetland microcosms. Proc. Biochem. 42, 363-373.

Inamori, R., Wang, Y., Yamamoto, T., Zhang, J., Kong, H., Xu, K., Inamori, Y., 2008. Seasonal effect on $\mathrm{N}_{2} \mathrm{O}$ formation in nitrification in constructed wetlands. Chemosphere 73 (7), 1071-1077.

IPCC, 2006. IPCC guidelines for national greenhouse gas inventories. Agriculture, Forestry and other Land Use, vol. 4. Institute for Global Environmental Strategies (IGES), on behalf of the IPCC, Japan. 
IPCC., 2007. Climate Change: The Physical Science Basis. Cambridge University Press, Cambridge.

Itokawa, H., Hanaki, K., Matsuo, T., 2001. Nitrous oxide production in high-loading biological nitrogen removal process under low $\mathrm{COD} / \mathrm{N}$ ratio condition. Water Res. 35, 657-664.

Jäckel, U., Schnell, S., 2000. Suppression of methane emission from rice paddies by ferric iron fertilization. Soil Biol. Biochem. 32, 1811-1814.

Jia, W., Zhang, J., Li, P., Xie, H., Wu, J., Wang, J., 2011. Nitrous oxide emissions from surface flow and subsurface flow constructed wetland microcosms: effect of feeding strategies. Ecol. Eng. 37 (11), 1815-1821.

Johansson, A.E., Klemedtsson, A.K., Klemedtsson, L., Svensson, B.H., 2003. Nitrous oxide exchanges with the atmosphere of a constructed wetland treating wastewater - parameters and implications for emission factors. Tellus B 55 (3), 737-750.

Johansson, A.E., Gustavsson, A.M., Öquist, M.G., Svensson, B.H., 2004. Methane emissions from a constructed wetland treating wastewater - seasonal and spatial distribution and dependence on edaphic factors. Water Res. 38 (18), 3960-3970.

Juutinen, S., Larmola, T., Remus, R., Mirus, E., Merbach, W., Silvola, J., Augustin, J., 2003. The contribution of Phragmites australis litter to methane $\left(\mathrm{CH}_{4}\right)$ emission in planted and non-planted fen microcosms. Biol. Fertil. Soils 38, 10-14.

Kadlec, R.H., Knight, R.L., 1996. Treatment Wetlands. CRC Press/Lewis Publishers, Boca Raton, FL, pp. 893.

Kadlec, R.H., Wallace, S.D., 2008. Treatment Wetlands, 2nd ed. CRC Press, Boca Raton, FL, pp. 1016

Kalbitz, K., Solinger, S., Park, J.H., Michalzik, B., Matzner, E., 2000. Controls on the dynamics of dissolved organic matter in soils: a review. Soil Sci. 165 (4), 277-304.

Kayranli, B., Scholz, M., Mustafa, A., Hedmark, A., 2010. Carbon storage and fluxes within freshwater wetlands: a critical review. Wetlands 30 (1), 111-124.

Klemedtsson, L., von Arnold, K., Weslien, P., Gundersen, P., 2005. Soil CN ratio as a scalar parameter to predict nitrous oxide emissions. Global Change Biol. 11, $1142-1147$

Knowles, R., 1982. Denitrification. Microbiol. Rev. 46 (1), 43-70.

Kumaraswamy, S., Ramakrishnan, B., Sethunathan, N., 2001. Methane production and oxidation in an anoxic rice soil as influenced by inorganic redox species. J. Environ. Qual. 30, 2195-2201.

Le Mer, J., Roger, P., 2001. Production, oxidation, emission and consumption of methane by soils: a review. Eur. J. Soil Biol. 37 (1), 25-50

Ligi, T., Oopkaup, K., Truu, M., Preem, J.-K., Nõlvak, H., Mitsch, W.J., Mander, Ü., Truu, J., 2014a. Characterisation of bacterial communities in soil and sediment of a created riverine wetland complex using high-throughput 16S rRNA amplicon sequencing. Ecol. Eng, http://dx.doi.org/10.1016/j.ecoleng.2013.09.007 (in press).

Ligi, T., Truu, M., Truu, J., Nõlvak, H., Kaasik, A., Mitsch, W.J., Mander, Ü., 2014b. Effects of soil chemical characteristics and water regime on denitrification genes (nirS, nirK, and nosZ) abundances in a created riverine wetland complex. Ecol. Eng., http://dx.doi.org/10.1016/j.ecoleng.2013.07.015 (in press).

Liikanen, A., Huttunen, J.T., Karjalainen, S.M., Heikkinen, K., Väisänen, T.S., Nykänen, H., Martikainen, P.J., 2006. Temporal and seasonal changes in greenhouse gas emissions from a constructed wetland purifying peat mining runoff waters. Ecol. Eng. 26 (3), 241-251.

Liu, C., Xu, K., Inamori, R., Ebie, Y., Liao, J., Inamori, Y., 2009. Pilot-scale studies of domestic wastewater treatment by typical constructed wetlands and their greenhouse gas emissions. Front. Environ. Sci. Eng. China 3 (4), 477-482.

Maddison, M., Mauring, T., Remm, K., Lesta, M., Mander, Ü., 2009. Dynamics of Typha latifolia L. populations in treatment wetlands in Estonia. Ecol. Eng. 35 (2), 258-264.

Maltais-Landry, G., Maranger, R., Brisson, J., 2009a. Effect of artificial aeration and macrophyte species on nitrogen cycling and gas flux in constructed wetlands. Ecol. Eng. 35 (2), 221-229.

Maltais-Landry, G., Maranger, R., Brisson, J., Chazarenc, F., 2009b. Greenhouse gas production and efficiency of planted and artificially aerated constructed wetlands. Environ. Pollut. 157 (3), 748-754.

Mander, Ü., Jenssen, P.D. (Eds.), 2003. Constructed Wetlands for Wastewater Treatment in Cold Climates. WIT Press, Southampton, UK, p. 325

Mander, Ü., Kuusemets, V., Lõhmus, K., Mauring, T., Teiter, S., Augustin, J., 2003. Nitrous oxide, dinitrogen, and methane emission in a subsurface flow constructed wetland. Water Sci. Technol. 48 (5), 135-142.

Mander, Ü., Lõhmus, K., Teiter, S., Nurk, K., Mauring, T., Augustin, J., 2005a. Gaseous fluxes from subsurface flow constructed wetlands for wastewater treatment. J. Environ. Sci. Health A 40 (6/7), 1215-1226.

Mander, Ü., Teiter, S., Augustin, J., 2005b. Emission of greenhouse gases from constructed wetlands for wastewater treatment and from riparian buffer zones. Water Sci. Technol. 52 (11/12), 167-176.

Mander, Ü., Lõhmus, K., Teiter, S., Mauring, T., Nurk, K., Augustin, J., 2008. Gaseous fluxes in the nitrogen and carbon budgets of subsurface flow constructed wetlands. Sci. Total Environ. 404, 343-353.

Mander, Ü., Maddison, M., Soosaar, K., Karabelnik, K., 2011. The impact of intermittent hydrology and fluctuating water table on greenhouse gas emissions from subsurface flow constructed wetlands for wastewater treatment. Wetlands 31 (6), 1023-1032

Mander, Ü., Tournebize, J., Kasak, K., Mitsch, W.J., 2014. Climate regulation by free water surface constructed wetlands for wastewater treatment and created riverine wetlands. Ecol. Eng., http://dx.doi.org/10.1016/j.ecoleng.2013.05.004 (in press).
Mata-Alvarez, J., Macé, S., Llabrés, P., 2000. Anaerobic digestion of organic solid wastes: an overview of research achievements and perspectives. Bioresour. Technol 74 (1), 3-16.

Meerburg, B.G., Vereijken, P.H., de Visser, W., Korevaar, H., Querner, E.P., Blaeij, A.T. van der Werf, A.K., 2010. Surface water sanitation and biomass production in large constructed wetland in the Netherlands. Wetlands Ecol. Manage. 18 (4), 463-470.

Meijide, A., Cardenas, L.M., Bol, R., Bergstermann, A., Goulding, K., Well, R., Vallejo, A., Scholefield, D., 2010. Dual isotope and isotopomer measurements for the understanding of $\mathrm{N}_{2} \mathrm{O}$ production and consumption during denitrification in an arable soil. Eur. J. Soil Sci. 61, 364-374.

Mitsch, W.J., Gosselink, J.G., 2007. Wetlands. John Wiley and Sons, Hoboken, NJ, USA pp. 582.

Mitsch, W.J., Bernal, B., Nahlik, A.M., Mander, Ü., Zhang, L., Anderson, L., Jørgensen, S.E., Brix, H., 2013. Wetlands, carbon, and climate change. Landscape Ecol. 28 (4), 583-597.

Mosier, A.R., 1998. Soil processes and global change. Biol. Fertil. Soils 27, 221-229.

Mosier, A., Kroeze, C., Nevison, C., Oenema, O., Seitzinger, S., Van Cleemput, O., 1998 Closing the global $\mathrm{N}_{2} \mathrm{O}$ budget: nitrous oxide emissions through the agricultural nitrogen cycle. OECD/IPCC/IEA phase II development of IPCC guidelines for national greenhouse gas inventory methodology. Nutr. Cycl. Agroecosyst. 52, $225-248$.

Pan, T., Zhu, X.D., Ye, Y.P., 2011. Estimate of life-cycle greenhouse gas emissions from a vertical subsurface flow constructed wetland and conventional wastewater treatment plants: a case study in China. Ecol. Eng. 37 (2), 248-254.

Pangala, S.R., Reay, D.S., Heal, K.V., 2009. Mitigation of methane emissions from constructed farm wetlands. Chemosphere 78 (5), 493-499.

Park, K.Y., Inamori, Y., Mizuochi, M., Ahn, K.H., 2000. Emission and control of nitrous oxide from a biological wastewater treatment system with intermittent aeration. J. Biosci. Bioeng. 90, 247-252.

Park, K.Y., Lee, J.W., Inamori, Y., Mizuochi, M., Ahn, K.H., 2001. Effects of fill modes on $\mathrm{N}_{2} \mathrm{O}$ emission from the SBR treating domestic wastewater. Water Sci. Technol. 43, 147-150.

Picek, T., Čižkova, H., Dušek, J., 2007. Greenhouse gas emissions from a constructed wetland - plants as important sources of carbon. Ecol. Eng. 31 (2), 98-106.

Pulou, J., (Ph.D. Thesis) 2011. Les anciennes cressonnières de l'Essonne: effets de la recolonisation des zones humides artificielles sur la dynamique de l'azote. AgroParisTech, pp. 212

Riya, S., Zhou, S., Nakashimada, Y., Terada, A., Hosomi, M., 2010. Direct and indirect greenhouse gas emissions from vertical flow constructed wetland planted with forage rice. Kagaku Kogaku Ronbunshu 36 (4), 229-236.

Robertson, G.P., Tiedje, J.M., 1987. Nitrous oxide sources in aerobic soils: nitrification, denitrification and other biological processes. Soil Biol. Biochem. 19 (2) $187-193$.

Rückauf, U., Augustin, J., Russow, R., Merbach, W., 2004. Nitrate removal from drained and reflooded fen soils affected by soil $\mathrm{N}$ transformation processes and plant uptake. Soil Biol. Biochem. 36, 77-90.

Schafer, C.-M., Elsgaard, L., Hoffmann, C.C., Petersen, S.O., 2012. Seasonal methane dynamics in three temperate grasslands on peat. Plant Soil 357 (1/2), 339-353.

Seitzinger, S.P., Kroeze, C., 1998. Global distribution of nitrous oxide production and $\mathrm{N}$ inputs in freshwater and coastal marine ecosystems. Global Biogeochem. Cycle 12, 93-113

Sha, C.Y., Mitsch, W.J., Mander, Ü., Lu, J.J., Batson, J., Zhang, L., He, W.S., 2011. Methane emissions from freshwater riverine wetlands. Ecol. Eng. 37 (1), 16-24.

Silvan, N., Tuittila, E.S., Kitunen, V., Vasander, H., Laine, J., 2005. Nitrate uptake by Eriophorum vaginatum controls $\mathrm{N}_{2} \mathrm{O}$ production in a restored peatland. Soil Biol. Biochem. 37 (8), 1519-1526.

Smialek, J., Bouchard, V., Lippmann, B., Quigley, M., Granata, T., Martin, J., Brown, L., 2006. Effect of a woody (Salix nigra) and an herbaceous (Juncus effusus) macrophyte species on methane dynamics and denitrification. Wetlands 26 (2), 509-517.

Song, K., Kang, H., Zhang, L., Mitsch, W.J., 2012. Seasonal and spatial variations of denitrification and denitrifying bacterial community structure in created riverine wetlands. Ecol. Eng. 38 (1), 130-134.

Søvik, A.K., Kløve, B., 2007. Emission of $\mathrm{N}_{2} \mathrm{O}$ and $\mathrm{CH}_{4}$ from a constructed wetland in southeastern Norway. Sci. Total Environ. 380, 28-37.

Søvik, A.K., Augustin, J., Heikkinen, K., Huttunen, J.T., Necki, J.M., Karjalainen, S.M., Kløve, B., Liikanen, A., Mander, Ü., Puustinen, M., Teiter, 2S., Wachniew, P., 2006. Emission of the greenhouse gases nitrous oxide and methane from constructed wetlands in Europe. J. Environ. Qual. 35 (6), 2360-2373.

Spieles, D.J., Mitsch, W.J., 2000. The effects of season and hydrologic and chemica loading on nitrate retention in constructed wetlands: a comparison of low- and high-nutrient riverine systems. Ecol. Eng. 14 (1/2), 77-91.

Stadmark, J., Leonardson, L., 2005. Emissions of greenhouse gases from ponds constructed for nitrogen removal. Ecol. Eng. 25 (5), 542-551.

Ström, L., Mastepanov, M., Christensen, T.R., 2005. Species-specific effects of vascula plants on carbon turnover and methane emissions from wetlands. Biogeochemistry 75 (1), 65-82.

Ström, L., Lamppa, A., Christensen, T.R., 2006. Greenhouse gas emissions from a constructed wetland in southern Sweden. Wetlands Ecol. Manage. 15 (1), 43-50.

Tai, P.D., Li, P.J., Sun, T.H., He, Y.W., Zhou, Q.X., Gong, Z.Q., Mizuochi, M., Inamori, Y., 2002. Greenhouse gas emissions from a constructed wetland for municipal sewage treatment. J. Environ. Sci. China 14 (1), 27-33.

Tanner, C.C., Adams, D.D., Downes, M.T., 1997. Methane emissions from constructed wetlands treating agricultural wastewaters. J. Environ. Qual. 26 (4), 1056-1062. 
Teiter, S., Mander, Ü., 2005. Emission of $\mathrm{N}_{2} \mathrm{O}, \mathrm{N}_{2}, \mathrm{CH}_{4}$ and $\mathrm{CO}_{2}$ from constructed wetlands for wastewater treatment and from riparian buffer zones. Ecol. Eng. 25 (5), 528-541.

Thiere, G., Stadmark, J., Weisner, S.E.B., 2011. Nitrogen retention versus methane emission: environmental benefits and risks of large-scale wetland creation. Ecol. Eng. 37 (1), 6-15.

Tonderski, K., Hansson, A., 2001. Reduktion av kväve i våtmarker - pilotförsök vid Nykvarnsverket, Linköping (Nitrogen removal in wetlands - pilot scale experiments at Nykvarn, Linköping). Final Report. Tekniska Verken i Linköping AB, Linköping (in Swedish).

UKWIR, 2008. Carbon Accounting in the UK Water Industry: Operational Emissions. Report Reference No. 08/CL/01/5. UK Water Industry Research Limited, London.

Van de Riet, B.P., Hefting, M.M., Verhoeven, J.T.A., 2013. Rewetting drained peat meadows: risks and benefits in terms of nutrient release and greenhouse gas exchange. Water Air Soil Pollut. 224, 1440-1452.

Van der Gon, H.A.C.D., Neue, H.U., 1994. Impact of gypsum application on the methane emission from a wetland rice field. Global Biogeochem. Cycle 8 $127-134$

Van der Zaag, A.C., Gordon, R.J., Burton, D.L., Jamieson, R.C., Stratton, G.W. 2010. Greenhouse gas emissions from surface flow and subsurface flow constructed wetlands treating dairy wastewater. J. Environ. Qual. 39 (2), 460471.

Vilain, G., Garnier, J., Tallec, G., Cellier, P., 2010. Effect of slope position and land use on nitrous oxide $\left(\mathrm{N}_{2} \mathrm{O}\right)$ emissions (Seine Basin, France). Agric. Forest Meteorol. $150,1192-1202$

Vilain, G., Garnier, J., Tallec, G., Tournebize, J., 2012. Indirect $\mathrm{N}_{2} \mathrm{O}$ emissions from shallow groundwater in an agricultural catchment (Seine Basin, France). Biogeochemistry 111 (1-3), 253-271.

Vohla, C., Kõiv, M., Bavor, H.J., Chazarenc, F., Mander, Ü., 2011. Filter materials fo phosphorus removal from wastewater in treatment wetlands - a review. Ecol. Eng. 37 (1), 70-89.

Vymazal, J., 2001. Types of constructed wetlands for wastewater treatment: their potential for nutrient removal. In: Vymazal, J. (Ed.). Transformations of Nutrients in Natural and Constructed Wetlands. Backhuys Publishers, Leiden, The Netherlands, pp. 1-93.

Vymazal, J., 2007. Removal of nutrients in various types of constructed wetlands. Sci. Total Environ. 380, 48-65.

Vymazal, J., 2011. Constructed wetlands for wastewater treatment: five decades of experience. Environ. Sci. Technol. 45 (1), 65-69.

Vymazal, J., Kröpfelova, L., 2008. Wastewater Treatment in Constructed Wetlands with Horizontal Sub-Surface Flow. Springer, Dordrecht, pp. 566.

Vymazal, J., Brix, H., Cooper, P.F., Haberl, R., Perfler, R., Laber, J., 1998. Removal mechanisms and types of constructed wetlands. In: Vymazal, J., Brix, H., Cooper, P.F. Green, M.B., Haberl, R. (Eds.), Constructed Wetlands for Wastewater Treatment in Europe. Backhuys Publishers, Leiden, The Netherlands, pp. 17-66.

Waddington, J.M., Roulet, N.T.,Swanson, R.V., 1996. Water table control of $\mathrm{CH}_{4}$ emis sion enhancement by vascular plants in boreal peatlands. J. Geophys. Res. Atmos. 101 (D17), 22775-22785

Wang, Z.P., Delaune, R.D., Patrick Jr., W.H., Masscheleyn, P.H., 1993. Soil redox and $\mathrm{pH}$ effects on methane production in a flooded rice soils. Soil Sci. Soc. Am. J. 57 382-385.
Wang, Y., Inamori, R., Kong, H., Xu, K., Inamori, Y., Kondo, T., Zhang, J., 2008a. Influence of plant species and wastewater strength on constructed wetland methane emissions and microbial populations. Ecol. Eng. 32, 22-29.

Wang, Y., Inamori, R., Kong, H., Xu, K., Inamori, Y., Kondo, T., Zhang, J., 2008b. Nitrous oxide emission from polyculture constructed wetlands: effect of plant species. Environ. Pollut. 152 (2), 351-360.

Well, R., Weymann, D., Flessa, H., 2005. Recent research progress on the significance of aquatic systems for indirect agricultural $\mathrm{N}_{2} \mathrm{O}$ emissions. Environ. Sci. 2 (2/3), $143-151$.

Well, R., Eschenbach, W., Flessa, H., von der Heide, C., Weymann, D., 2012. Are dual isotope and isotopomer ratios of $\mathrm{N}_{2} \mathrm{O}$ useful indicators for $\mathrm{N}_{2} \mathrm{O}$ turnover during denitrification in nitrate-contaminated aquifers? Geochim. Cosmochim. Acta 90, 265-282

Wild, U., Kamp, T., Lenz, A., Heinz, S., Pfadenhauer, J., 2001. Cultivation of Typha spp. in constructed wetlands for peatland restoration. Ecol. Eng. 17 (1), 49-54

Williams, H.G., Bialowiec, A., Slater, F., Randerson, P.F., 2010. Spatial variation of dissolved gas concentrations in a willow vegetation filter treating landfill leachate. Ecol. Eng. 36 (12), 1774-1778.

Willison, T.W., Baker, J.C., Murphy, D.V., 1998. Methane fluxes and nitrogen dynamics from a drained fenland peat. Biol. Fertil. Soils 27 (3), 279-283.

Wrage, N., Velthof, G.L., van Beusichem, M.L., Oenema, O., 2001. Role of nitrifier denitrification in the production of nitrous oxide. Soil Biol. Biochem. 33, 1723-1732.

Wu, J., Zhang, J., Jia, W.L., Xie, H.J., Gu, R.R., Li, C., Gao, B., 2009. Impact of COD/N ratio on nitrous oxide emission from microcosm wetlands and their performance in removing nitrogen from wastewater. Bioresour. Technol. 100 (12), 2910-2917.

Wunderlin, P., Lehmann, M.F., Siegrist, H., Tuzson, B., Joss, A., Emmenegger, L., Mohn, J., 2013. Isotope signatures of $\mathrm{N}_{2} \mathrm{O}$ in a mixed microbial population system: constraints on $\mathrm{N}_{2} \mathrm{O}$ producing pathways in wastewater treatment. Environ. Sci. Technol. 47 (3), 1339-1348.

Xue, Y., Kovacic, D.A., David, M.B., Gentry, L.E., Mulvaney, R.L., Lindau, C.W., 1999 In situ measurements of denitrification in constructed wetlands. J. Environ. Qual. 28 (1), 263-269

Yan, C., Zhang, H., Li, B., Wang, D., Zhao, Y., Zheng, Z., 2012. Effects of influent C/N ratios on $\mathrm{CO}_{2}$ and $\mathrm{CH}_{4}$ emissions from vertical subsurface flow constructed wetlands treating synthetic municipal wastewater. J. Hazard. Mater. 203/204, $188-194$.

Yang, J., Liu, J., Hu, X., Li, X., Wang, Y., Li, H., 2013. Effect of water table level on $\mathrm{CO}_{2}, \mathrm{CH}_{4}$ and $\mathrm{N}_{2} \mathrm{O}$ emissions in a freshwater marsh of Northeast China. Soil Biol. Biochem. 61, 52-60.

Yu, K.W., Faulkner, S.P., Patrick, W.H., 2006. Redox potential characterization and soil greenhouse gas concentration across a hydrological gradient in a Gulf coast forest. Chemosphere 62 (6), 905-914.

Zhou, S., Hou, H., Hosomi, M., 2008. Nitrogen removal, $\mathrm{N}_{2} \mathrm{O}$ emission, and $\mathrm{NH}_{3}$ volatilization under different water levels in a vertical flow treatment system. Water Air Soil Pollut. 191 (1-4), 171-182.

Zhu, N.W., An, P., Krishnakumar, B., Zhao, L., Sun, L., Mizuochi, M., Inamori, Y., 2007. Effect of plant harvest on methane emission from two constructed wetlands designed for the treatment of wastewater. J. Environ. Manage. 85 (4), 936-943. 